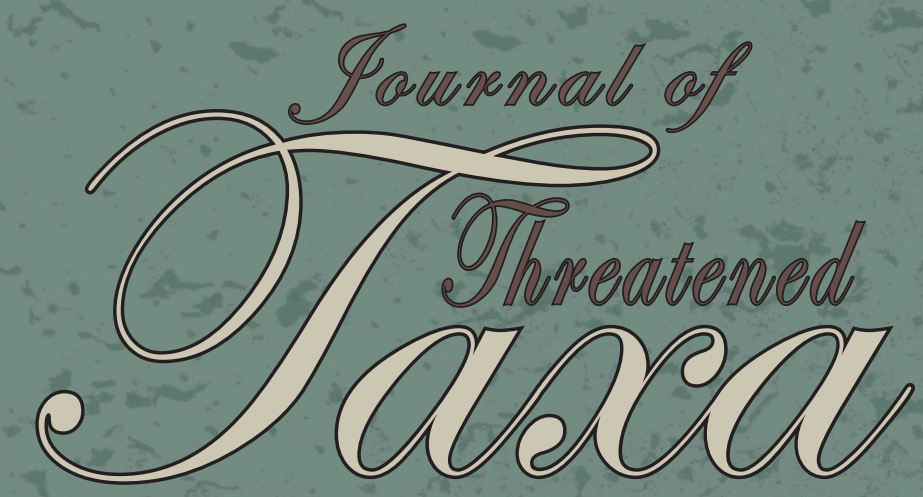

Building evidence for conservation globally
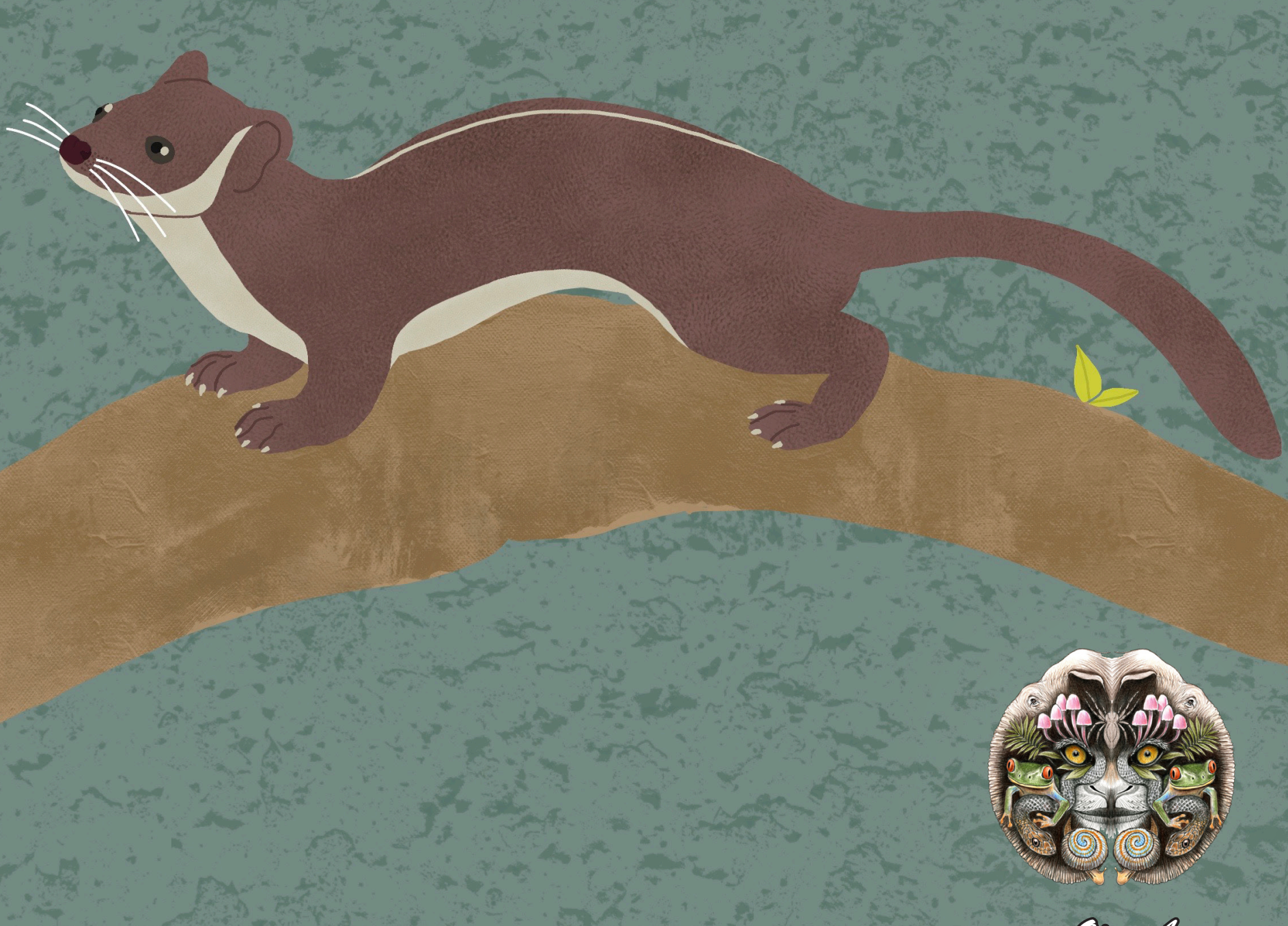

Qpecosecess

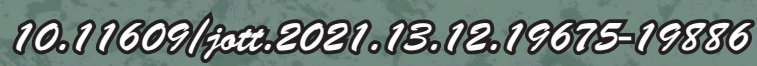
cocosurthreatecredtassararg

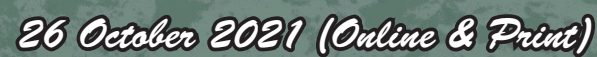

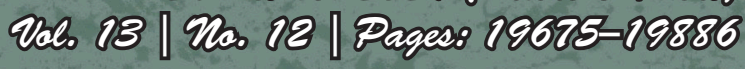




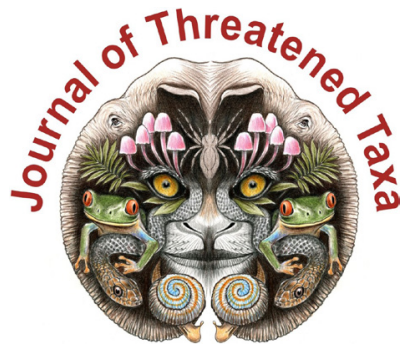

ISSN 0974-7907 (Online); ISSN $0974-7893$ (Print)

Publisher

Host

Wildlife Information Liaison Development Society

www.wild.zooreach.org

Zoo Outreach Organization www.zooreach.org

No. 12, Thiruvannamalai Nagar, Saravanampatti - Kalapatti Road, Saravanampatti, Coimbatore, Tamil Nadu 641035, India

Ph: +91 9385339863 | www.threatenedtaxa.org

Email: sanjay@threatenedtaxa.org

EDITORS

\section{Founder \& Chief Editor}

Dr. Sanjay Molur

Wildlife Information Liaison Development (WILD) Society \& Zoo Outreach Organization (ZOO)

12 Thiruvannamalai Nagar, Saravanampatti, Coimbatore, Tamil Nadu 641035, India

\section{Deputy Chief Edito}

Dr. Neelesh Dahanukar

Noida, Uttar Pradesh, India

\section{Managing Editor}

Mr. B. Ravichandran, WILD/ZOO, Coimbatore, India

\section{Associate Editors}

Dr. Mandar Paingankar, Government Science College Gadchiroli, Maharashtra 442605, India Dr. Ulrike Streicher, Wildlife Veterinarian, Eugene, Oregon, USA

Ms. Priyanka Iyer, ZOO/WILD, Coimbatore, Tamil Nadu 641035, India

Dr. B. A. Daniel, $200 /$ WIID, Coimbatore, Tamil Nadu 641035, India

\section{Editorial Board}

Dr. Russel Mittermeie

Executive Vice Chair, Conservation International, Arlington, Virginia 22202, USA

\section{Prof. Mewa Singh Ph.D., FASc, FNA, FNASc, FNAPsy}

Ramanna Fellow and Life-Long Distinguished Professor, Biopsychology Laboratory, and Institute of Excellence, University of Mysore, Mysuru, Karnataka 570006, India; Honorary Professor, Jawaharlal Nehru Centre for Advanced Scientific Research, Bangalore; and Adjunct Professor, National Institute of Advanced Studies, Bangalore

\section{Stephen D. Nash}

Scientific Illustrator, Conservation International, Dept. of Anatomical Sciences, Health Sciences Center, T-8, Room 045, Stony Brook University, Stony Brook, NY 11794-8081, USA

\section{Dr. Fred Pluthero}

Toronto, Canada

\section{Dr. Priya Davidar}

Sigur Nature Trust, Chadapatti, Mavinhalla PO, Nilgiris, Tamil Nadu 643223, India

\section{Dr. Martin Fisher}

Senior Associate Professor, Battcock Centre for Experimental Astrophysics, Cavendish

Laboratory, JJ Thomson Avenue, Cambridge CB3 OHE, UK

\section{Dr. John Fellowes}

Honorary Assistant Professor, The Kadoorie Institute, 8/F, T.T. Tsui Building, The University of Hong Kong, Pokfulam Road, Hong Kong

\section{Prof. Dr. Mirco Solé}

Universidade Estadual de Santa Cruz, Departamento de Ciências Biológicas, Vice-coordenado do Programa de Pós-Graduação em Zoologia, Rodovia Ilhéus/Itabuna, Km 16 (45662-000)

Salobrinho, Ilhéus - Bahia - Brasil

\section{Dr. Rajeev Raghavan}

Professor of Taxonomy, Kerala University of Fisheries \& Ocean Studies, Kochi, Kerala, India

\section{English Editors}

Mrs. Mira Bhojwani, Pune, India

Dr. Fred Pluthero, Toronto, Canad

Mr. P. Ilangovan, Chennai, India

Web Maintenance

Mrs. Latha G. Ravikumar, ZOO/WILD, Coimbatore, India

\section{Typesetting}

Mr. Arul Jagadish, ZOO, Coimbatore, India

Mrs. Radhika, ZOO, Coimbatore, India

Mrs. Geetha, ZOO, Coimbatore India

\section{Fundraising/Communications}

Mrs. Payal B. Molur, Coimbatore, India

Subject Editors 2018-2020

Fungi

Dr. B. Shivaraju, Bengaluru, Karnataka, India

Dr. R.K. Verma, Tropical Forest Research Institute, Jabalpur, India

Dr. Vatsavaya S. Raju, Kakatiay University, Warangal, Andhra Pradesh, India

Dr. M. Krishnappa, Jnana Sahyadri, Kuvempu University, Shimoga, Karnataka, India

Dr. K.R. Sridhar, Mangalore University, Mangalagangotri, Mangalore, Karnataka, India

Dr. Gunjan Biswas, Vidyasagar University, Midnapore, West Bengal, India

Plants

Dr. G.P. Sinha, Botanical Survey of India, Allahabad, India

Dr. N.P. Balakrishnan, Ret. Joint Director, BSI, Coimbatore, India

Dr. Shonil Bhagwat, Open University and University of Oxford, UK

Prof. D.J. Bhat, Retd. Professor, Goa University, Goa, India

Dr. Ferdinando Boero, Università del Salento, Lecce, Italy

Dr. Dale R. Calder, Royal Ontaro Museum, Toronto, Ontario, Canada

Dr. Cleofas Cervancia, Univ. of Philippines Los Baños College Laguna, Philippines

Dr. F.B. Vincent Florens, University of Mauritius, Mauritius

Dr. Merlin Franco, Curtin University, Malaysia

Dr. V. Irudayaraj, St. Xavier's College, Palayamkottai, Tamil Nadu, India

Dr. B.S. Kholia, Botanical Survey of India, Gangtok, Sikkim, India

Dr. Pankaj Kumar, Kadoorie Farm and Botanic Garden Corporation, Hong Kong S.A.R., China

Dr. V. Sampath Kumar, Botanical Survey of India, Howrah, West Bengal, India

Dr. A.J. Solomon Raju, Andhra University, Visakhapatnam, India

Dr. Vijayasankar Raman, University of Mississippi, USA

Dr. B. Ravi Prasad Rao, Sri Krishnadevaraya University, Anantpur, India

Dr. K. Ravikumar, FRLHT, Bengaluru, Karnataka, India

Dr. Aparna Watve, Pune, Maharashtra, India

Dr. Qiang Liu, Xishuangbanna Tropical Botanical Garden, Yunnan, China

Dr. Noor Azhar Mohamed Shazili, Universiti Malaysia Terengganu, Kuala Terengganu, Malaysia Dr. M.K. Vasudeva Rao, Shiv Ranjani Housing Society, Pune, Maharashtra, India

Prof. A.J. Solomon Raju, Andhra University, Visakhapatnam, India

Dr. Mandar Datar, Agharkar Research Institute, Pune, Maharashtra, India

Dr. M.K. Janarthanam, Goa University, Goa, India

Dr. K. Karthigeyan, Botanical Survey of India, India

Dr. Errol Vela, University of Montpellier, Montpellier, France

Dr. P. Lakshminarasimhan, Botanical Survey of India, Howrah, India

Dr. Larry R. Noblick, Montgomery Botanical Center, Miami, USA

Dr. K. Haridasan, Pallavur, Palakkad District, Kerala, India

Dr. Analinda Manila-Fajard, University of the Philippines Los Banos, Laguna, Philippines

Dr. P.A. Sinu, Central University of Kerala, Kasaragod, Kerala, India

Dr. Afroz Alam, Banasthali Vidyapith (accredited A grade by NAAC), Rajasthan, India

Dr. K.P. Rajesh, Zamorin's Guruvayurappan College, GA College PO, Kozhikode, Kerala, India

Dr. David E. Boufford, Harvard University Herbaria, Cambridge, MA 02138-2020, USA

Dr. Ritesh Kumar Choudhary, Agharkar Research Institute, Pune, Maharashtra, India

Dr. Navendu Page, Wildlife Institute of India, Chandrabani, Dehradun, Uttarakhand, India

Invertebrates

Dr. R.K. Avasthi, Rohtak University, Haryana, India

Dr. D.B. Bastawade, Maharashtra, India

Dr. Partha Pratim Bhattacharjee, Tripura University, Suryamaninagar, India

Dr. Kailash Chandra, Zoological Survey of India, Jabalpur, Madhya Pradesh, India

Dr. Ansie Dippenaar-Schoeman, University of Pretoria, Queenswood, South Africa

Dr. Rory Dow, National Museum of natural History Naturalis, The Netherlands

Dr. Brian Fisher, California Academy of Sciences, USA

Dr. Richard Gallon, llandudno, North Wales, LL30 1UP

Dr. Hemant V. Ghate, Modern College, Pune, India

Dr. M. Monwar Hossain, Jahangirnagar University, Dhaka, Bangladesh

Mr. Jatishwor Singh Irungbam, Biology Centre CAS, Branišovská, Czech Republic.

Dr. lan J. Kitching, Natural History Museum, Cromwell Road, UK

Dr. George Mathew, Kerala Forest Research Institute, Peechi, India

Dr. John Noyes, Natural History Museum, London, UK

For Focus, Scope, Aims, and Policies, visit https://threatenedtaxa.org/index.php/JoTT/aims_scope
For Article Submission Guidelines, visit https://threatenedtaxa.org/index.php/JoTT/about/submissions
For Policies against Scientific Misconduct, visit https://threatenedtaxa.org/index.php/JoTT/policies_various

continued on the back inside cover 


\title{
Checklist of moths (Heterocera) of Tadong, Sikkim, India
}

\author{
Prayash Chettri $^{1} \mathbb{D}$, Yuki Matsui ${ }^{2}$ (D), Hideshi Naka ${ }^{3}$ (D) \& Archana Tiwari $^{4}$ (D) \\ ${ }^{1}$ Department of Zoology, Darjeeling Government College, Darjeeling, West Bengal 734101, India. \\ ${ }^{2,3}$ Department of Agriculture, Tottori University, Tottori 680-8550, Tottori, Japan. \\ ${ }^{4}$ Department of Physics, Sikkim University, Gangtok, Sikkim 737102, India. \\ ${ }^{4}$ Department of Physics, Banaras Hindu University, Varanasi, Uttar Pradesh 221005, India. \\ ${ }^{1}$ prayashchettri96@gmail.com, ${ }^{2}$ mothya22@gmail.com, ${ }^{3}$ chun@tottori-u.ac.jp, ${ }^{4}$ archana.tiwari.ox@gmail.com (corresponding author)
}

\begin{abstract}
This study attempts to create a checklist of moths recorded from two different parts of Tadong in Sikkim, located in the northeastern Himalaya of India. Out of 160 photographed specimens of moths, 133 species were identified and classified. Sixteen families of moths were recorded out of which Erebidae (30.83\%) had the highest number of species followed by Geometridae (24.81\%), and Crambidae (18.05\%) while the other families comprised of $26.30 \%$ of the total species.
\end{abstract}

Keywords: Biodiversity, Eastern Himalaya, Erebidae, Geometridae.

Sikkim, a northeastern Himalayan state, is known for its biodiversity and strategic location surrounded by Nepal, Bhutan, and China. Several new species of butterflies, insects, and birds have been identified in the region (Kalawate 2018), but there are few reports on the sighting or identification of moth species. They play important roles in ecosystems as pollinators for many plants, and they are food for many predators, including bats and birds (Scoble 1992).

During $19^{\text {th }}-20^{\text {th }}$ Century, Hampson $(1892,1894$, 1895, 1896) and Bell \& Scott (1973) documented moths of this region. Dudgeon (1898-1901) documented moths from Sikkim and Bhutan. Kirti \& Sodhi (2002) recorded 30 species of footman moths from Sikkim.
The State Fauna Series holds records of moths from Ctenuchidae and Limacodidae, with 24 species and 40 species, respectively (Chaudhury 2003), Saturniidae with 26 species (Gupta 2003), Zygaenidae with 66 species (Bhattacharya 2003), Arctiidae with 182 species (Chaudhury 2003), and Geometridae with 265 species (Ghosh 2003). Kirti \& Sodhi (2003) recoded 24 species belonging to subfamily Arctiinae from Sikkim. Sanyal et al. (2018) recorded 4,107 species with Sikkim having the greatest moth diversity. Chandra et al. (2019) also recorded 1,274 species of moths in 'Assemblages of Lepidoptera in Indian Himalaya through Long Term Monitoring Plots,' where many of the species of moths were recorded from the state of Sikkim.

The aim of the present study is to create a baseline checklist of moths from Tadong region in Sikkim for further update and addition to the inventory of moths of Sikkim.

\section{MATERIALS AND MEthOdS}

An opportunistic survey was conducted where moths were photographed as they came towards light sources (LED, incandescent or compact fluorescent bulbs) illuminating residential premises. They were

Copyright: (c) Chettri et al. 2021. Creative Commons Attribution 4.0 International License. JoTT allows unrestricted use, reproduction, and distribution of this article in any medium by providing adequate credit to the author(s) and the source of publication.

Funding: Self-funded.

Competing interests: The authors declare no competing interests.

Acknowledgements: AT would like to express sincere gratitude to Prof. Sudipta Mukherji, Institute of Physics, Bhubaneswar, Dr. Ajay Tripathi, Sikkim University and Ms. Tavishi Tripathi, Varanasi for their constant motivation, support and encouragement. PC would like to express his sincere gratitude to Mr. Anthony Chettri for his constant support and encouragement and also for providing the basic needs for the accomplishment of this study. 
photographed in two different localities in Tadong (Figure 1a-c) viz., Gairi Gaon (27.314N \& 88.601E) and $6^{\text {th }}$ Mile area $(27.3127 \mathrm{~N} \& 88.593 \mathrm{E})$ within the period of 2017-2019. The altitude of the sites ranged from 1,0991,356 m (Table 1). Study sites are located between two watercourses, Rani Khola and Rorochu. The region has taken on urban characteristics as land resources have been encroached continuously due rapid unplanned development (Figure 1d) (Chettri \& Lama 2014). There is a gradual change in vegetation from alpine to subtropical and temperate deciduous forest in the region (Tamang et al. 2005). The temperature has been increasing in recent decades and precipitation fluctuates each year (Kumar et al. 2020).

The survey included photographing moths near the light sources using a smartphone camera (12MP).
Table 1. Geographical location of survey sites at Tadong, Sikkim, India.

\begin{tabular}{|l|l|c|}
\hline Survey Sites & GPS Coordinates & Altitude $(\mathbf{m})$ \\
\hline Gairi Gaon & $27.314 \mathrm{~N} \& 88.601 \mathrm{E}$ & 1,099 \\
\hline $6^{\text {th }}$ Mile & $27.312 \mathrm{~N} \& 88.593 \mathrm{E}$ & 1,356 \\
\hline
\end{tabular}

Morphological characters were considered for the identification. Different sources were used including Walker (1866), Haruta (1992-2000), Irungbam et al. (2016), Shubhalaxmi (2018), Kirti \& Singh (2015), Sondhi \& Sondhi (2016), and Uniyal et al. (2016). Online portals such as Indian Biodiversity Portal (Vattakaven et al. 2016), iNaturalist, LepIndex (Beccaloni et al. 2003), and BOLDSYSTEMS (Ratnasingham \& Hebert 2007) were also utilised for the identification. Classification has been

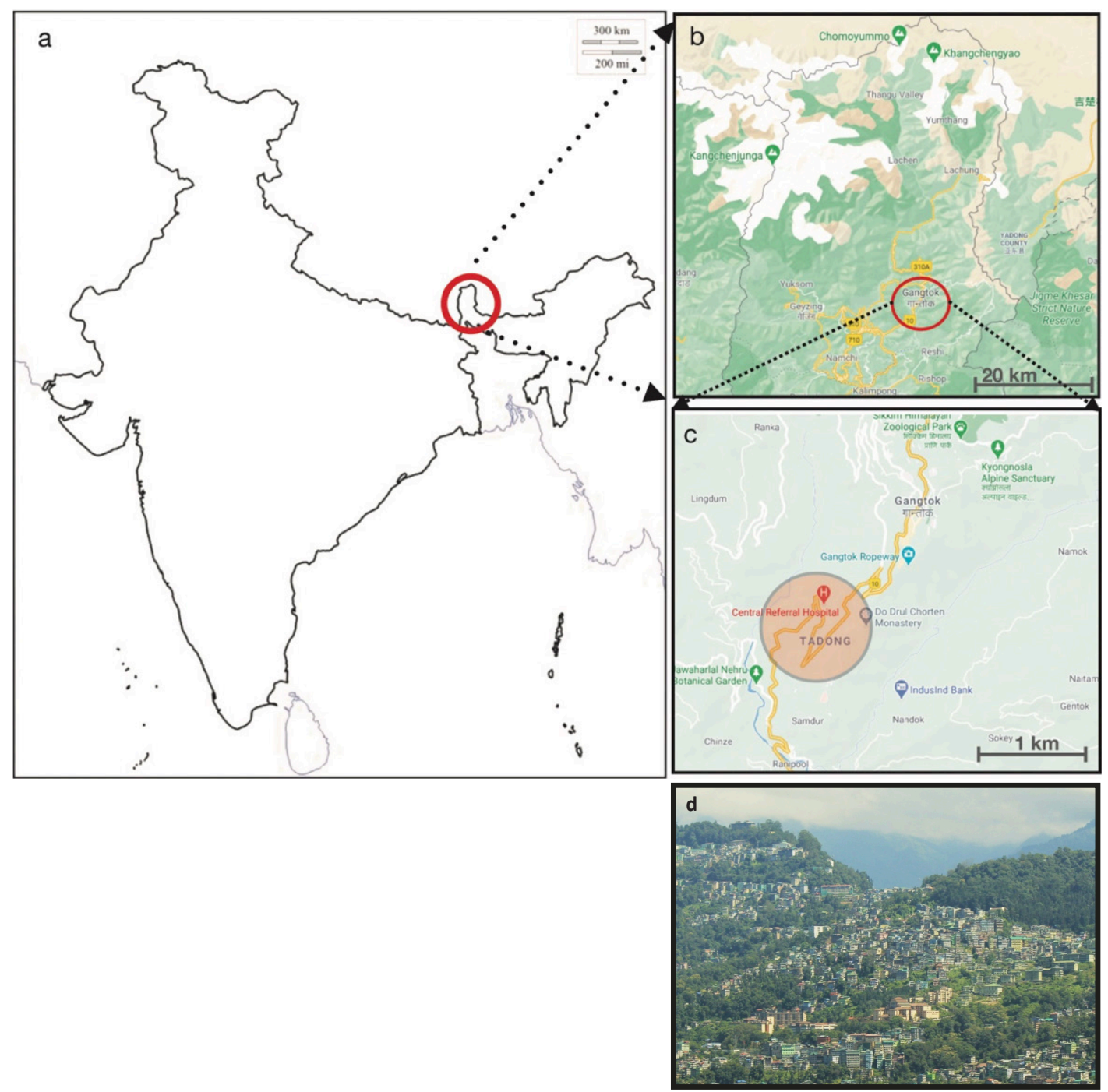

Figure 1. Location of survey site: $a-$ Border region where the survey sites were located with respect to the map | b-Magnified scale indicating topography within the geographical boundary | c-GPS beacon showing approximate location of survey sites (Gairi Gaon and $6^{\text {th }}$ Mile), Tadong, Sikkim | d-Close-up view of the study sites showing human settlement encroached landscape. 
followed according to van Nieukerken et al. (2011) and Nuss et al. (2003-2021). PAST3 (Computer Software) was used to calculate diversity indices. Species contribution to diversity of each family was determined by calculating the dominance index $=\mathrm{ni} \times 100 / \mathrm{N}$ where $(\mathrm{ni})$ is individuals of particular species and $(\mathrm{N})$ is the total no. of species (Mishra et al. 2016).

\section{RESULTS AND DISCUSSION}

During the study a total of 160 individual moths were photographed, from which 133 species were identified from the region. Of the identified specimens 112 were identified to species level, and 21 to the genus level (Images 1-133). Looking at species contribution to the diversity it was found that family Erebidae had the highest number of moths with 41 species, followed by Geometridae with 33 species and Crambidae with 24 species. Other families including Noctuidae, Notodontidae, Pyralidae, Drepanidae, Eupterotidae, Nolidae, Zygaenidae, Bombycidae, Limacodidae, Lasiocampidae, and Thyrididae accounted for $26 \%$ of total species (Table 2, Figure 2). All the identified species are listed in Table 3. Diversity indices were calculated using Past3 software, which showed Fisher's alpha, Shannon index, evenness, and Chao-1 to be 4.752, $1.975,0.4504$, and 21 , respectively. Hence, the species diversity seems quite high. These values could be later used to collate species diversity (abundance, richness,

Table 2. Number of moth species belonging to different families.

\begin{tabular}{|c|c|c|}
\hline & Family & No. of species \\
\hline 1 & Erebidae & 41 \\
\hline 2 & Geometridae & 33 \\
\hline 3 & Crambidae & 24 \\
\hline 4 & Noctuidae & 7 \\
\hline 5 & Nolidae & 5 \\
\hline 6 & Notodontidae & 5 \\
\hline 7 & Pyralidae & 5 \\
\hline 8 & Drepanidae & 3 \\
\hline 9 & Eupterotidae & 2 \\
\hline 10 & Zyganidae & 2 \\
\hline 11 & Bombycidae & 1 \\
\hline 12 & Euteliidae & 1 \\
\hline 13 & Limacodidae & 1 \\
\hline 14 & Lasiocampidae & 1 \\
\hline 15 & Thyrididae & 1 \\
\hline 16 & Tortricidae & 1 \\
\hline \multicolumn{2}{|c|}{ Total } & 133 \\
\hline
\end{tabular}

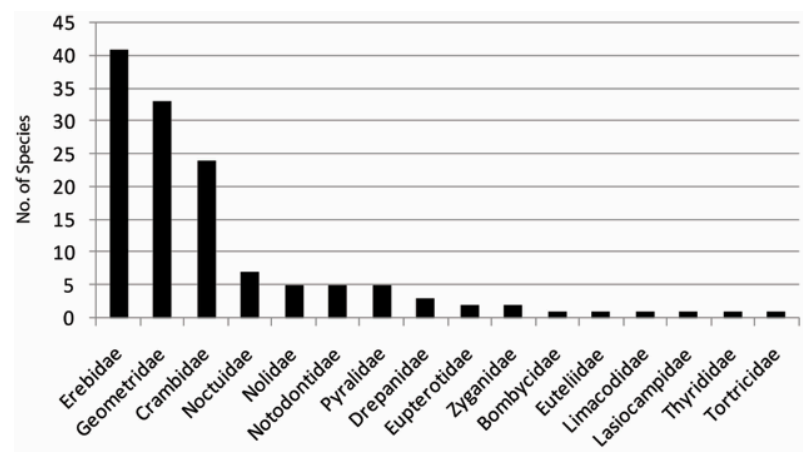

Figure 3. Histogram showing distribution of number of identified species belonging to different families.

evenness) of moths of this region.

\section{CONCLUSION}

In this study, we have attempted to create a baseline checklist of moths from Tadong, Sikkim region. This work adds to the inventory of moths of this region which could be utilised for future studies.

\section{REFERENCES}

Beccaloni, G., M. Scoble, I. Kitching, T. Simonsen, G. Robinson, B. Pitkin, A. Hine \& C. Lyal (eds.) (2003). The Global Lepidoptera Names Index (Leplndex). World Wide Web electronic publication. https://www.nhm.ac.uk/our-science/data/lepindex/lepindex/ Accessed 12 November 2020]

Bell, T.R.D \& F.B. Scott (1937) .The Fauna of British India, including Ceylon and Burma, Moths. Sphingidae. Taylor \& Francis Ltd., London, 537pp.

Bhattacharya, D.P. (2003). Insecta: Lepidoptera: Zyganidae. State Fauna Series 9, Fauna of Sikkim (Part-4): 159-174.

Chandra, K., V. Kumar, N. Singh, A. Raha, and A.K. Sanyal (2019). Assemblages of Lepidoptera in Indian Himalaya through Long Term Monitoring Plots. Zoological Survey of India, 457pp.

Chaudhury, M.M. (2003). Insecta: Lepidoptera: Arctiidae. State Fauna Series 9, Fauna of Sikkim (Part-4): 175-216.

Chaudhury, M.M. (2003). Insecta: Lepidoptera: Ctenuchidae and Limacodidae. State Fauna Series 9, Fauna of Sikkim (Part-4): 93-107.

Chettri, A. \& S. Lama (2014). Trends of urbanization and its impacts on environmental resources: A case study of Gangtok town of Sikkim Himalayas. Geo-Analyst, 76-85pp.

Dudgeon, G.C. (1898). A catalogue of the Heterocera of Sikkim and Bhutan, part 1. Journal of the Bombay Natural History Society 11(1): 239-251.

Dudgeon, G.C. (1899). A catalogue of the Heterocera of Sikkim and Bhutan, part 5. Journal of the Bombay Natural History Society 12: 292-303.

Dudgeon, G.C. (1901). A catalogue of the Heterocera of Sikkim and Bhutan, part 2. Journal of the Bombay Natural History Society 11(2): 406-419.

Ghosh, S.K. (2003). Insecta: Lepidoptera: Heterocera: Geometridae. State Fauna Series 9, Fauna of Sikkim (Part-4): 217-342.

Gupta, I.J. (2003). Insecta: Lepidoptera: Saturnidae. State Fauna Series 9. Fauna of Sikkim (Part-4): 147-158.

Hampson, G.F. (1892). The Fauna of British India, including Ceylon and Burma, Moths. Vol 1, Saturniidae to Hypsiade. Taylor \& Francis, London, 527pp. 
Table 3. Checklist of moths recorded during this survey.

\begin{tabular}{|c|c|c|c|c|c|c|}
\hline & Family & Subfamily & Scientific name & $\begin{array}{l}\text { Author and Year of } \\
\text { description }\end{array}$ & $\begin{array}{l}\text { Months observed } \\
\text { (2017-2019) }\end{array}$ & Location \\
\hline 1 & Bombycidae & Bombycinae & Penicillifera apicalis & Walker, 1862 & September & GG \\
\hline 2 & Crambidae & Acentropinae & Eristena sp. & & June & SM \\
\hline 3 & Crambidae & Acentropinae & Parapoynx fluctuosalis & Meyrick, 1899 & August, June & SM \\
\hline 4 & Crambidae & Odontiinae & Heortia vitessoides & Moore, 1885 & May & SM \\
\hline 5 & Crambidae & Pyraustinae & Hyalobathra coenostolalis & Snellen, 1890 & October & SM \\
\hline 6 & Crambidae & Pyraustinae & Pagyda auroralis & Moore, 1888 & September & GG \\
\hline 7 & Crambidae & Pyraustinae & Sclerocona sp. & & October & SM \\
\hline 8 & Crambidae & Spilomelinae & Agrotera basinotata & Hampson, 1891 & June & SM \\
\hline 9 & Crambidae & Spilomelinae & Arthroschista hilaralis & Walker, 1859 & August & SM \\
\hline 10 & Crambidae & Spilomelinae & Bradina diagonalis & Guenée, 1854 & November & GG \\
\hline 11 & Crambidae & Spilomelinae & Cnaphalocrocis medinalis & Guenée, 1854 & October & GG \\
\hline 12 & Crambidae & Spilomelinae & Diaphania indica & Saunders, 1851 & June & SM \\
\hline 13 & Crambidae & Spilomelinae & Glyphodes crithealis & Walker, 1859 & May & GG \\
\hline 14 & Crambidae & Spilomelinae & Leucinodes orbonalis & Guenée, 1854 & July & SM \\
\hline 15 & Crambidae & Spilomelinae & Cnaphalocrocis trapizalis & Guenée, 1854 & November & SM \\
\hline 16 & Crambidae & Spilomelinae & Maruca vitrata & Fabricius, 1787 & October & GG \\
\hline 17 & Crambidae & Spilomelinae & Metoeca foedalis & Guenée, 1854 & November & GG \\
\hline 18 & Crambidae & Spilomelinae & Patania scinisalis & Walker, 1859 & September & GG \\
\hline 19 & Crambidae & Spilomelinae & Perisyntrocha ossealis & Hampson, 1896 & October & GG \\
\hline 20 & Crambidae & Spilomelinae & Pycnarmon aeriferalis & Moore, 1877 & November & SM \\
\hline 21 & Crambidae & Spilomelinae & Pygospila tyres & Cramer, 1780 & March, August & GG \\
\hline 22 & Crambidae & Spilomelinae & Rhimphalea trogusalis & Walker, 1859 & July & SM,GG \\
\hline 23 & Crambidae & Spilomelinae & Spoladae recurvalis & Fabricius, 1775 & August & GG \\
\hline 24 & Crambidae & Spilomelinae & Synclera cf. univocalis & Walker, 1859 & August & SM \\
\hline 25 & Crambidae & Spilomelinae & Talanga sp. & & August & GG \\
\hline 26 & Drepanidae & Drepaninae & Callidrepana sp. & & September & SM \\
\hline 27 & Drepanidae & Drepaninae & Drepana pallida & Warren, 1922 & $\begin{array}{l}\text { November, } \\
\text { October }\end{array}$ & GG \\
\hline 28 & Drepanidae & Drepaninae & Macrocilix maia & Leech, 1888 & October & GG \\
\hline 29 & Erebidae & Aganainae & Asota caricae & Fabricius, 1775 & May & SM \\
\hline 30 & Erebidae & Aganainae & Asota plana & Walker, 1854 & April & GG,SM \\
\hline 31 & Erebidae & Aganainae & Mecodina cineracea & Butler, 1879 & September & SM \\
\hline 32 & Erebidae & Arctiinae & Adites frigida & Walker, 1854 & July & SM \\
\hline 33 & Erebidae & Arctiinae & Aemene taprobanis & Walker, 1854 & October & GG \\
\hline 34 & Erebidae & Arctiinae & Aglaomorpha plagiata & Walker, 1855 & March & GG \\
\hline 35 & Erebidae & Arctiinae & Barsine cf. cuneonotata & Walker, 1855 & July & GG \\
\hline 36 & Erebidae & Arctiinae & Indiania eccentropis & Meyrick, 1894 & May & GG \\
\hline 37 & Erebidae & Arctiinae & Camptoloma binotatum & Butler, 1881 & June & SM \\
\hline 38 & Erebidae & Arctiinae & Creatonotos transiens & Walker, 1855 & June & SM \\
\hline 39 & Erebidae & Arctiinae & Cyana cf. coccinea & Moore, 1878 & October & SM \\
\hline 40 & Erebidae & Arctiinae & Cyana cf. neopuer & Singh et al. 2019 & August & GG \\
\hline 41 & Erebidae & Arctiinae & Cyana cf. weerawoothi & Lourens, 2017 & October & SM \\
\hline 42 & Erebidae & Arctiinae & Lyclene cf. clamaria & Moore, 1888 & October & SM \\
\hline 43 & Erebidae & Arctiinae & Lyclene conjunctana & Walker, 1866 & July & GG \\
\hline 44 & Erebidae & Arctiinae & Lyclene dasara & Moore, 1859 & October & SM \\
\hline 45 & Erebidae & Arctiinae & Barsine phaeodonta & Hampson, 1911 & October & GG \\
\hline
\end{tabular}




\begin{tabular}{|c|c|c|c|c|c|c|}
\hline & Family & Subfamily & Scientific name & $\begin{array}{l}\text { Author and Year of } \\
\text { description }\end{array}$ & $\begin{array}{l}\text { Months observed } \\
\text { (2017-2019) }\end{array}$ & Location \\
\hline 46 & Erebidae & Arctiinae & Nyctemera adversata & Schaller, 1788 & May & SM \\
\hline 47 & Erebidae & Arctiinae & Padenia duplicana & Walker, 1863 & August & SM \\
\hline 48 & Erebidae & Arctiinae & Zardara distorta & Moore, 1894 & June & SM \\
\hline 49 & Erebidae & Arctiinae & Pseudoblabes oophora & Zeller, 1853 & October & GG \\
\hline 50 & Erebidae & Arctiinae & Schistophleps bipuncta & Hampson, 1891 & October & GG \\
\hline 51 & Erebidae & Arctiinae & Juxtarxia multiguttata & Walker, 1855 & May, August & SM \\
\hline 52 & Erebidae & Arctiinae & Spilarctia sp. & & September & SM \\
\hline 53 & Erebidae & Arctiinae & Stictane sp. & & April & GG \\
\hline 54 & Erebidae & Arctiinae & Syntomoides imaon & Cramer, 1780 & October & SM \\
\hline 55 & Erebidae & Boletobiinae & Singara diversalis & Walker, 1865 & August & GG \\
\hline 56 & Erebidae & Calpinae & Eudocima sp. & & July & SM \\
\hline 57 & Erebidae & Erebinae & Anomis flava & Anomis flava & April & GG \\
\hline 58 & Erebidae & Erebinae & Artena dotata & Fabricius, 1794 & October & GG \\
\hline 59 & Erebidae & Erebinae & Erebus gemmans & Guenée, 1852 & September & GG \\
\hline 60 & Erebidae & Hypeninae & Dichromia quadralis & Walker, 1858 & November & GG \\
\hline 61 & Erebidae & Lymantriinae & Cifuna locuples & Walker, 1855 & May & GG \\
\hline 62 & Erebidae & Lymantriinae & Euproctis bipunctapex & Hampson, 1891 & May & GG \\
\hline 63 & Erebidae & Lymantriinae & Euproctis cf. postica & Walker 1865 & May & GG \\
\hline 64 & Erebidae & Lymantriinae & Euproctis sp. & & November & SM \\
\hline 65 & Erebidae & Lymantriinae & llema sp. & & July & GG \\
\hline 66 & Erebidae & Lymantriinae & Pida apicalis & Walker, 1865 & December & GG \\
\hline 67 & Erebidae & Lymantriinae & Somena scintillans & Walker, 1856 & May & SM \\
\hline 68 & Erebidae & Lymantriinae & Somena similis & Moore, 1860 & October & GG \\
\hline 69 & Erebidae & Pangraptinae & Pangrapta pseudalbistigma & Yoshimoto, 1993 & October & GG \\
\hline 70 & Eupterotidae & Eupterotinae & Apha sp. & & October & GG \\
\hline 71 & Eupterotidae & Eupterotinae & Eupterote geminata & Walker, 1855 & September & GG \\
\hline 72 & Euteliidae & Stictopterinae & Lophoptera squammigera & Guenée, 1852 & August & SM \\
\hline 73 & Geometridae & Desmobathrinae & Eumelea cf. atomata & & November & SM \\
\hline 74 & Geometridae & Ennominae & Abraxas neomartaria & Inoue, 1970 & November & GG \\
\hline 75 & Geometridae & Ennominae & Arichanna transfasciata & Warren, 1893 & May & SM \\
\hline 76 & Geometridae & Ennominae & Biston contectaria & Walker, 1863 & September & SM \\
\hline 77 & Geometridae & Ennominae & Cassyma cf. deletaria & Moore, 1888 & September & SM \\
\hline 78 & Geometridae & Ennominae & Celenna festivaria & Fabricius, 1794 & June & GG \\
\hline 79 & Geometridae & Ennominae & Chorodna mauraria & Guenée, 1858 & May & GG \\
\hline 80 & Geometridae & Ennominae & Cleora fraterna & Moore, 1888 & August, November & GG \\
\hline 81 & Geometridae & Ennominae & Cleorasp. & & August & GG \\
\hline 82 & Geometridae & Ennominae & Corymica immaculata & Warren, 1897 & October & GG \\
\hline 83 & Geometridae & Ennominae & Darisa fratercula & Moore, 1888 & March & GG \\
\hline 84 & Geometridae & Ennominae & Ectropis dentilineata & Moore, 1867 & May, June & SM \\
\hline 85 & Geometridae & Ennominae & Gonodontis aethocrypta & Prout, 1962 & November & SM \\
\hline 86 & Geometridae & Ennominae & Erebomorpha fulgurita & Walker, 1860 & September & GG \\
\hline 87 & Geometridae & Ennominae & Parasynegia pluristriaria & Walker, 1863 & September & GG \\
\hline 88 & Geometridae & Ennominae & Heterostegane subtessellata & Walker, 1862 & November & GG \\
\hline 89 & Geometridae & Ennominae & Ourapteryx clara & Butler, 1880 & October & SM \\
\hline 90 & Geometridae & Ennominae & Hypomecis sp. & & October & GG \\
\hline 91 & Geometridae & Ennominae & Thinopteryx crocoptera & Kollar, 1844 & April & GG \\
\hline
\end{tabular}




\begin{tabular}{|c|c|c|c|c|c|c|}
\hline & Family & Subfamily & Scientific name & $\begin{array}{l}\text { Author and Year of } \\
\text { description }\end{array}$ & $\begin{array}{l}\text { Months observed } \\
\text { (2017-2019) }\end{array}$ & Location \\
\hline 92 & Geometridae & Ennominae & Thinopteryx nebulosa & Butler, 1883 & October & SM \\
\hline 93 & Geometridae & Geometrinae & Comibaena integranota & Hampson, 1893 & September & SM \\
\hline 94 & Geometridae & Geometrinae & Cosmostolodes albicantena & Warren, 1895 & March & GG \\
\hline 95 & Geometridae & Geometrinae & Maxates cf. protrusa & Butler, 1878 & May & SM \\
\hline 96 & Geometridae & Geometrinae & Thalassodes quadraria & Guenée, 1857 & November & SM \\
\hline 97 & Geometridae & Larentiinae & Acolutha pictaria & Moore, 1888 & December & SM \\
\hline 98 & Geometridae & Larentiinae & Harutalcis cf. vialis & Moore, 1888 & November & SM \\
\hline 99 & Geometridae & Larentiinae & Syzeuxis sp. & & May & SM \\
\hline 100 & Geometridae & Larentiinae & Polynesia truncapex & Swinhoe, 1892 & July & GG \\
\hline 101 & Geometridae & Oenochrominae & Sarcinodes restitutaria & Walker, 1863 & August & GG \\
\hline 102 & Geometridae & Sterrhinae & Perixera absconditaria & Walker, 1862 & December & GG \\
\hline 103 & Geometridae & Sterrhinae & Scopula cf. ferrilineata & Moore, 1888 & November & GG \\
\hline 104 & Geometridae & Sterrhinae & Synegiodes histrionaria & Swinhoe, 1892 & March & SM \\
\hline 105 & Geometridae & Sterrhinae & Timandra correspondens & Hampson, 1895 & June & GG \\
\hline 106 & Lasiocampidae & Lasiocampinae & Trabala vishnou & Lefèbvre, 1827 & May, November & SM \\
\hline 107 & Limacodidae & Limacodinae & Chalcoscelides castaneipars & Moore, 1865 & August & SM \\
\hline 108 & Noctuidae & Aganainae & Cymatophoropsis sinuata & Moore, 1879 & May & SM \\
\hline 109 & Noctuidae & Catocalinae & Arcte modesta & Hoeven, 1840 & August & GG \\
\hline 110 & Noctuidae & Heliothinae & Heliothis peltigera & $\begin{array}{l}\text { Denis \& Schiffermüller, } \\
1775\end{array}$ & April & SM \\
\hline 111 & Noctuidae & Noctuinae & Mythimna intertexta & Chang, 1991 & June & SM \\
\hline 112 & Noctuidae & Noctuinae & Mythimna seperata & Walker, 1865 & April & GG \\
\hline 113 & Noctuidae & Noctuinae & Trachea auriplena & Walker, 1857 & April & GG \\
\hline 114 & Noctuidae & Plusiinae & Ctenoplusia agnata & Staudinger, 1892 & April & GG \\
\hline 115 & Nolidae & Chloephorinae & Gabala polyspilalis & Walker, 1865 & May & GG \\
\hline 116 & Nolidae & Chloephorinae & Kerala sp. & & May, October & GG \\
\hline 117 & Nolidae & Chloephorinae & Nycteola sp. & & October & GG \\
\hline 118 & Nolidae & Chloephorinae & Tyana cf. chloroleuca & Walker, 1866 & July & SM \\
\hline 119 & Nolidae & Risobinae & Risoba sp. & & November & GG \\
\hline 120 & Notodontidae & Cerurinae & Syntypistis pallidifascia & Hampson, 1892 & October & SM \\
\hline 121 & Notodontidae & Dudusinae & Netria multispinae & Schintlmeister, 2006 & July & GG \\
\hline 122 & Notodontidae & Phalerinae & Phalera grotei & Moore, 1859 & July & SM \\
\hline 123 & Notodontidae & Thaumetopoeinae & Gazalina chrysolopha & Kollar, 1844 & September & SM \\
\hline 124 & Notodontidae & Thaumetopoeinae & Gazalina transversa & Moore, 1879 & April & SM \\
\hline 125 & Pyralidae & Epipaschiinae & Orthaga sp. & & October & GG \\
\hline 126 & Pyralidae & Epipaschiinae & Salma sp. & & September & GG \\
\hline 127 & Pyralidae & Epipaschiinae & Teliphasa sp. & & September & GG \\
\hline 128 & Pyralidae & Pyralinae & Endotricha sp. & & April & GG \\
\hline 129 & Pyralidae & Pyralinae & Pyralis pictalis & Curtis, 1834 & September & GG \\
\hline 130 & Thyrididae & Striglininae & Telchines vialis & Moore, 1883 & May & GG \\
\hline 131 & Tortricidae & Tortricinae & Archips sp. & & July & GG \\
\hline 132 & Zygaenidae & Chalcosiinae & Agalope bifasciata & Hope, 1840 & August & GG \\
\hline 133 & Zygaenidae & Chalcosiinae & Pidorus glaucopis & Drury, 1773 & August & SM \\
\hline \multicolumn{7}{|c|}{$\begin{array}{l}\text { Note: 1) Survey sites: Gairi Gaon (GG) and } 6^{\text {th }} \text { Mile (SM); } 2 \text { ) Months observed: Includes months on which the species were sited which might indicate flying } \\
\text { duration or seasonal presence; 3) Scientific name: Genus level identifications are represented as sp. Provisional identifications as cf. or near, the former for close } \\
\text { matches and the latter for poor matches. }\end{array}$} \\
\hline
\end{tabular}



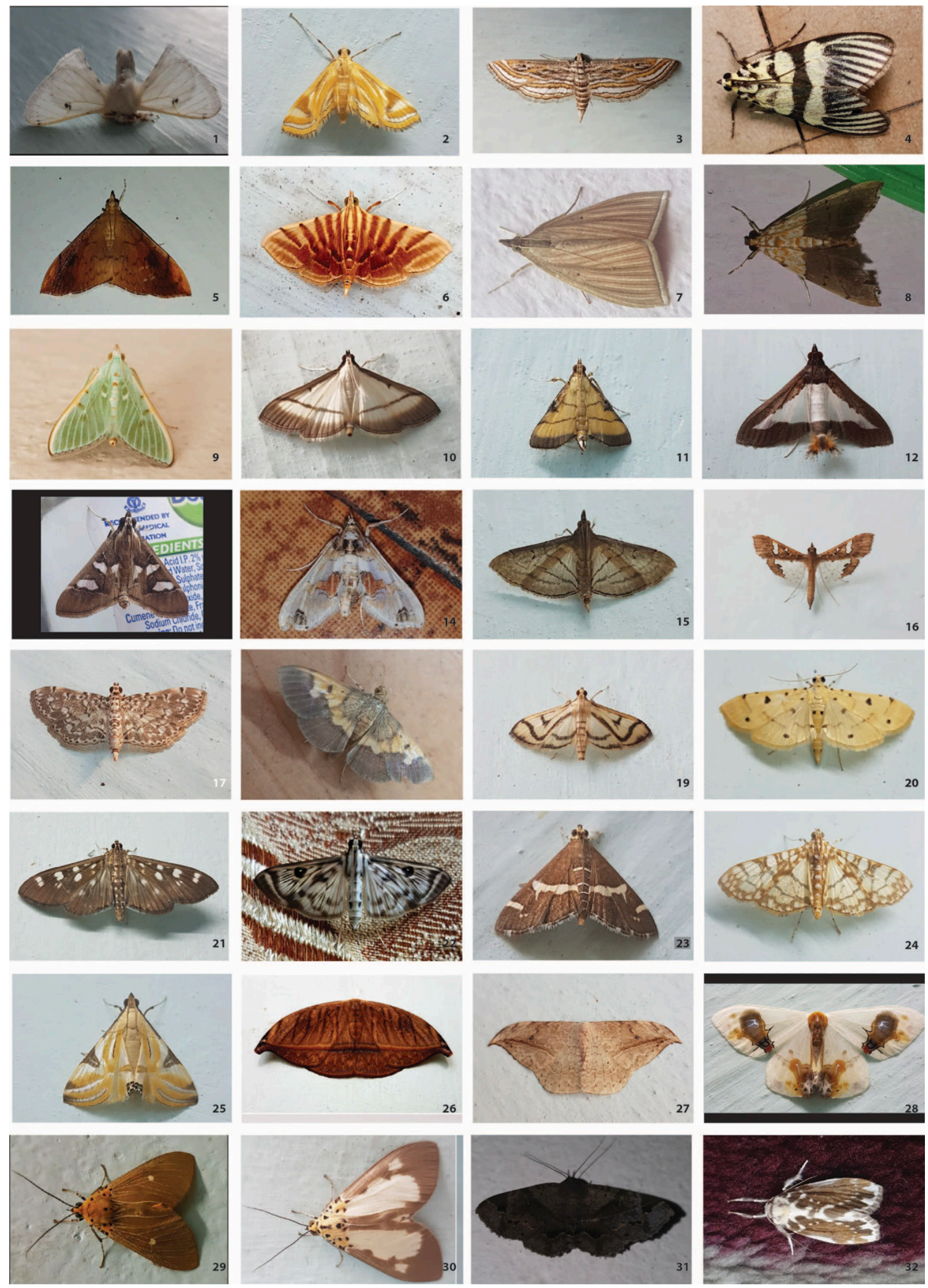

Image 1-Penicillifera apicalis | 2-Eristena sp. | 3-Parapoynx fluctuosalis | 4-Heortia vitessoides | 5-Hyalobathra coenostolalis | 6Pagyda auroralis | 7-Sclerocona sp. | 8-Agrotera basinotata | 9-Arthroschista hilaralis | 10-Bradina diagonalis | 11-Cnaphalocrocis medinalis | 12-Diaphania indica | 13-Glyphodes crithealis | 14-Leucinodes orbonalis | 15-Cnaphalocrocis trapizalis | 16-Maruca vitrata | 17-Metoeca foedalis | 18-Patania scinisalis | 19-Perisyntrocha ossealis | 20-Pycnarmon aeriferalis | 21-Pygospila tyres | 22Rhimphalea trogusalis | 23-Spoladae recurvalis | 24-Synclera cf. univocalis | 25-Talanga sp. | 26-Callidrepana sp. | 27-Drepana pallida | 28-Macrocilix maia | 29-Asota caricae | 30-Asota plana | 31-Mecodina cineracea | 32-Adites frigida | 33-Aemene taprobanis. 


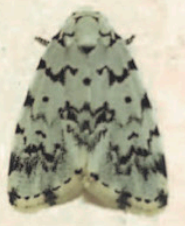

33
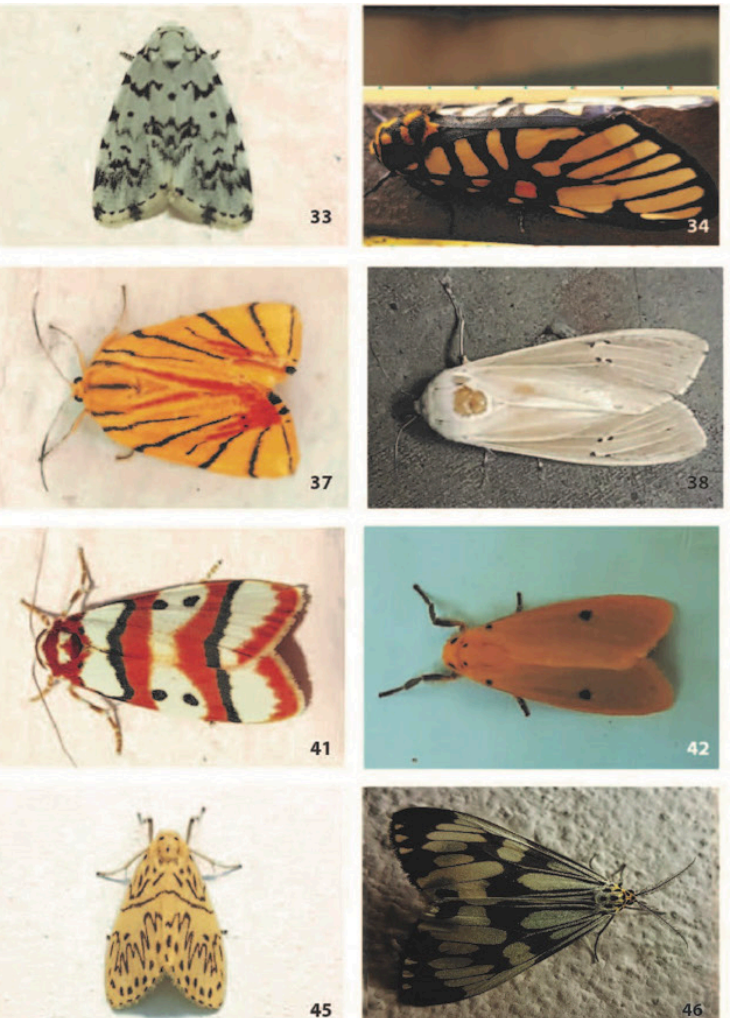

45
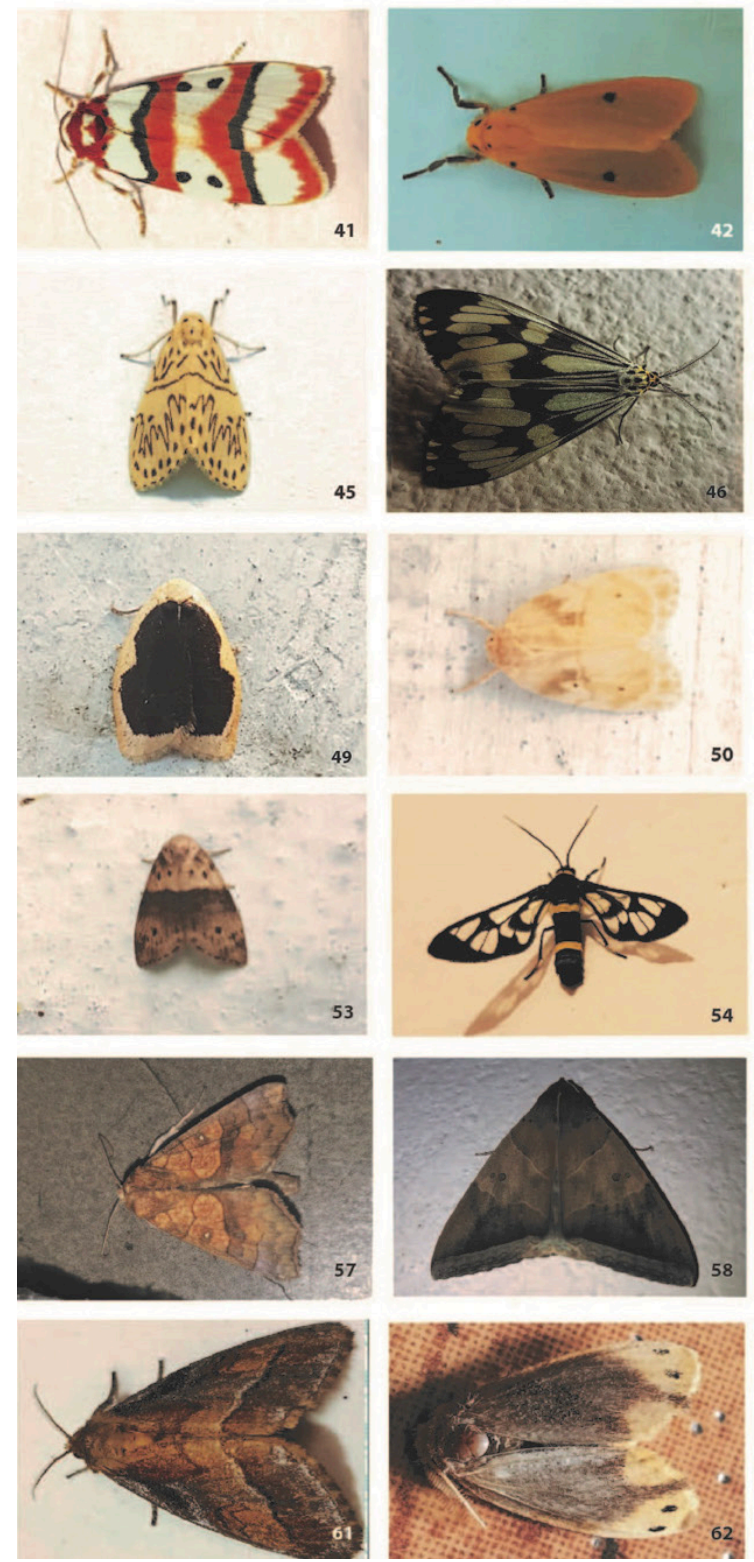
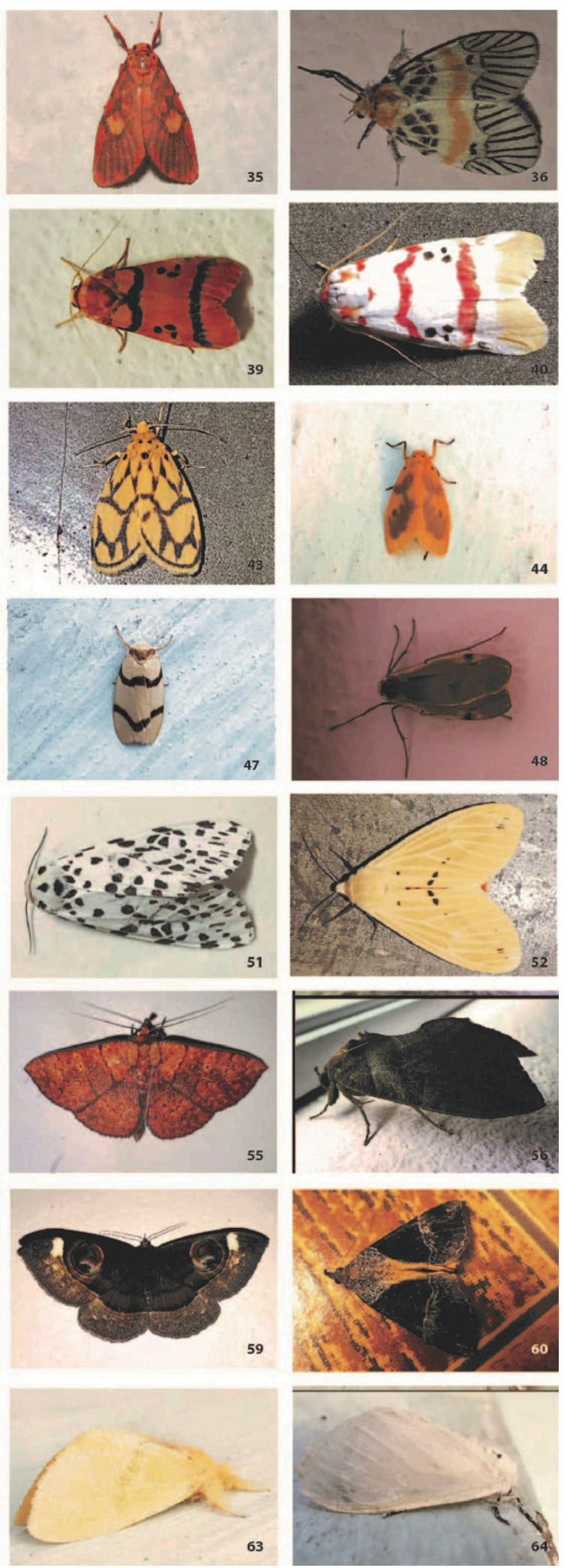

63

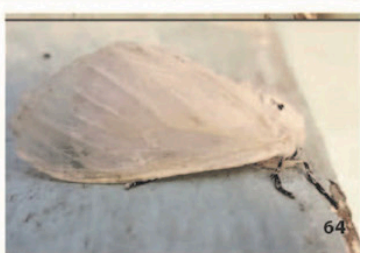

Image 34-Aglaomorpha plagiata | 35-Barsine cf. cuneonotata | 36-Indiania eccentropis | 37-Camptoloma binotatum | 38-Creatonotos transiens | 39-Cyana cf. coccinea | 40-Cyana cf. neopuer | 41-Cyana cf. weerawoothi | 42-Lyclene cf. clamaria | 43-Lyclene conjunctana | 44-Lyclene dasara | 45-Barsine phaeodonta | 46-Nyctemera adversata | 47-Padenia duplicana | 48-Zadadra distorta | 49Pseudoblabes oophora | 50-Schistophleps bipuncta | 51-Juxtarxia multiguttata | 52-Spilarctia sp. | 53-Stictane sp. | 54-Syntomoides imaon | 55-Singara diversalis | 56-Eudocima sp. | 57-Anomis flava | 58-Artena dotata | 59-Erebus gemmans | 60-Dichromia quadralis | 61-Cifuna locuples | 62-Euproctis bipunctapex | 63-Euproctis cf. postica | 64-Euproctis sp. | 65-Ilema sp. 

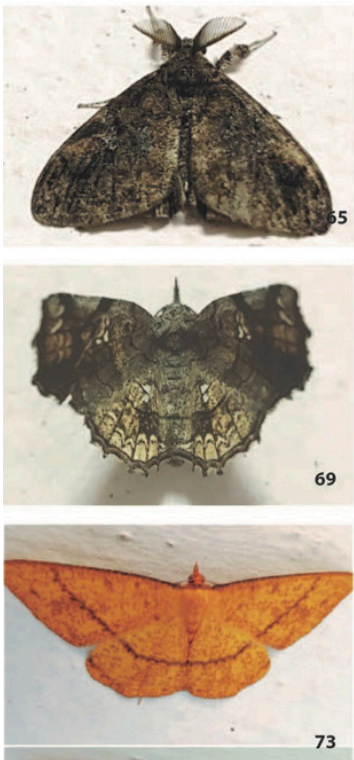

73

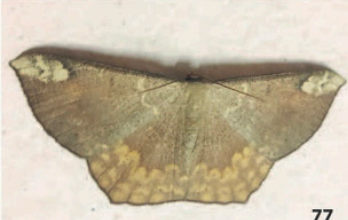

77
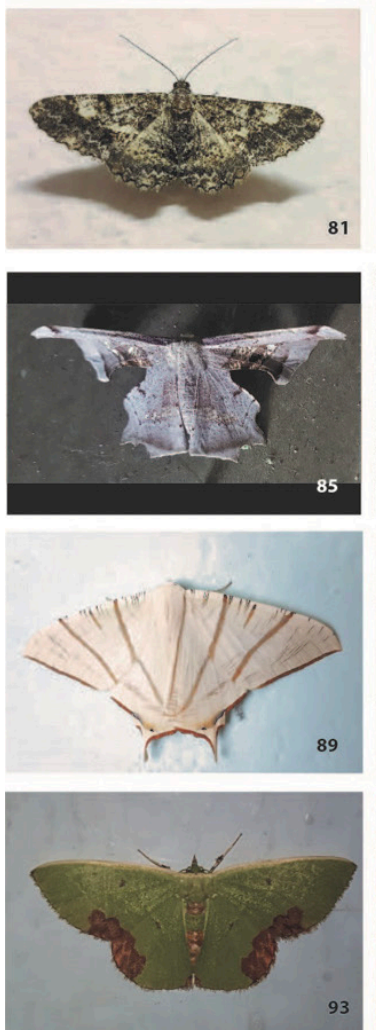

\section{(1)}
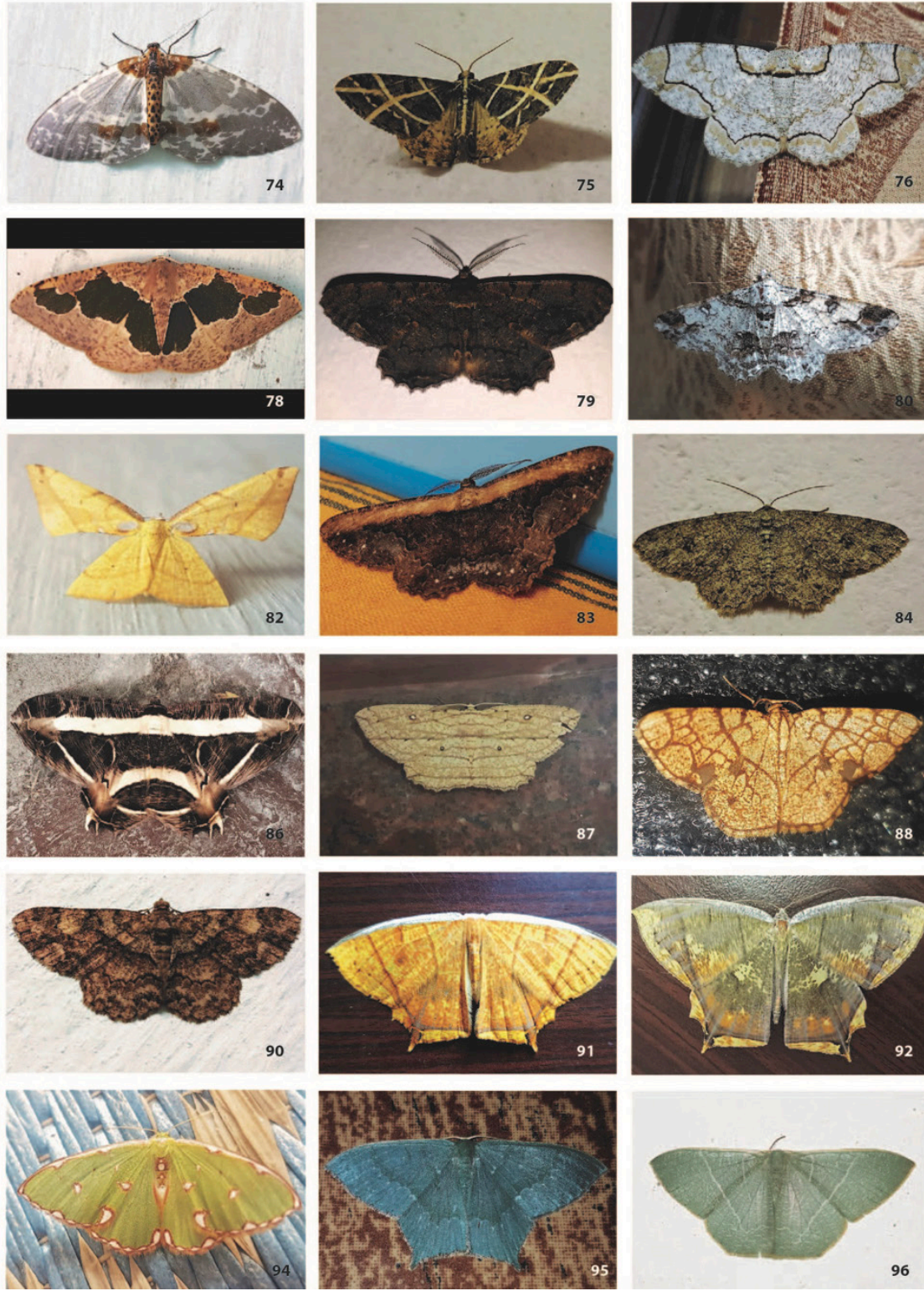

Images 66-97: 66-Pida apicalis | 67-Somena scintillans | 68-Somena similis | 69-Pangrapta pseudalbistigma | 70-Apha sp. | 71Eupterote cf. geminate | 72-Lophoptera squammigera | 73-Eumelea cf. atomata | 74-Abraxas neomartaria | 75-Arichanna transfasciata | 76-Biston contectaria | 77-Cassyma cf. deletaria | 78-Celenna festivaria | 79-Chorodna mauraria | 80-Cleora fraternal | 81-Cleora sp. | 82-Corymica immaculata | 83-Darisa fratercula | 84-Ectropis dentilineata | 85-Gonodontis aethocrypta | 86-Erebomorpha fulgurita | 87-Parasynegia pluristriaria | 88-Heterostegane subtessellata | 89-Ourapteryx clara | 90-Hypomecis sp. | 91-Thinopteryx crocoptera | 92-Thinopteryx nebulosa | 93-Comibaena integranota | 94-Cosmostolodes albicantena | 95-Maxates cf. protrusa | 96-Thalassodes quadraria | 97-Acolutha pictaria. 

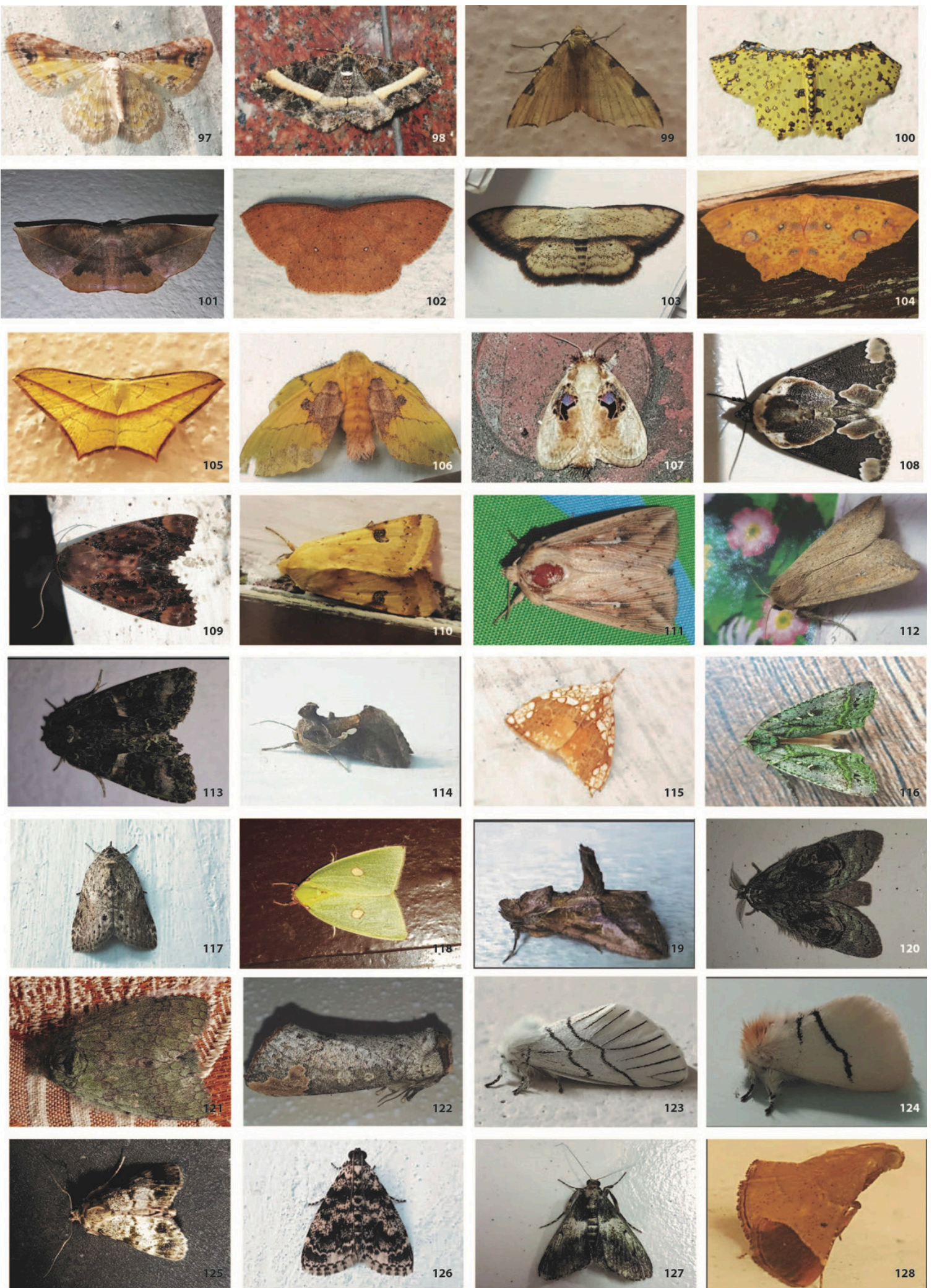

Image 98-Harutalcis cf. vialis | 99-Syzeuxis sp. | 100-Polynesia truncapex | 101-Sarcinodes restitutaria | 102-Perixera absconditaria | 103-Scopula cf. ferrilineata | 104-Synegiodes histrionaria | 105-Timandra correspondens | 106-Trabala vishnou | 107-Chalcoscelides castaneipars | 108-Cymatophoropsis sinuata | 109-Arcte modesta | 110-Heliothis peltigera | 111-Mythimna intertexta | 112-Mythimna seperata | 113-Trachea auriplena | 114-Ctenoplusia agnata | 115-Gabala polyspilalis | 116-Kerala sp. | 117-Nycteola sp. | 118-Tyana cf. chloroleuca | 119-Risoba sp. | 120-Syntypistis pallidifascia | 121-Netria multispinae | 122- Phalera grotei | 123-Gazalina chrysolopha | 124-Gazalina transversa | 125-Orthaga sp. | 126-Salsma sp. | 127-Teliphasa sp. | 128-Endotricha sp. | 129-Pyralis pictalis. 

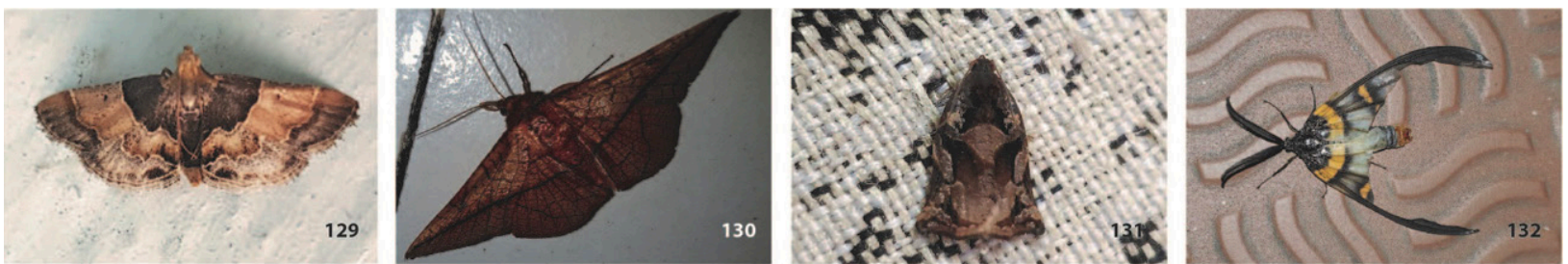

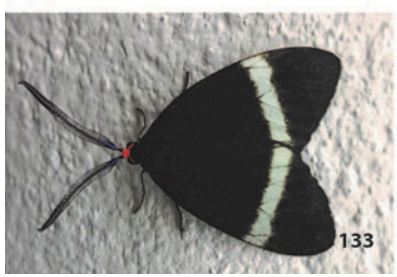

Images 130-133: 130-Telchines vialis | 131-Archips sp. | 132-Agalope bifasciata | 133-Pidorus glaucopis.

Hampson, G.F. (1894). The Fauna of British India, including Ceylon and Burma, Moths. Vol 2 Arctiidae, Agrostidae, Noctuidae. Taylor \& Francis, London, 609pp.

Hampson, G.F. (1895). The Fauna of British India, including Ceylon and Burma, Moths. Vol 3 Noctuidae (cont.) to Geometridae. Taylor \& Francis, London, 546pp.

Hampson, G.F. (1896). The Fauna of British India, including Ceylon and Burma, Moths. Vol 4 Pyralidae. Taylor \& Francis, London, 594pp.

Haruta, T. (Eds.) (1992). Moths of Nepal Part 1, Tinea 13 (Supplement 2). Japan Heterocerists' Society, Tokyo, 122pp.

Haruta, T. (Eds.) (1993). Moths of Nepal Part 2, Tinea 13 (Supplement 3). Japan Heterocerists' Society, Tokyo, 160pp.

Haruta, T. (Eds.) (1994). Moths of Nepal Part 3, Tinea 14 (Supplement 1). Japan Heterocerists' Society, Tokyo, 171pp.

Haruta, T. (Eds.) (1995). Moths of Nepal Part 4, Tinea 14 (Supplement 2). Japan Heterocerists' Society, Tokyo, 206pp.

Haruta, T. (Eds.) (1998). Moths of Nepal Part 5, Tinea 15(Supplement 1). Japan Heterocerists' Society, Tokyo, 330pp.

Haruta, T. (Eds.) (2000). Moths of Nepal Part 6, Tinea 16 (Supplement 1). Japan Heterocerists' Society, Tokyo, 163pp.

Irungbam, J.S., M.S. Chib \& K. Wangdi (2016). Taxonomic review of the superfamily Pyraloidea in Bhutan (Lepidoptera). Journal of Asia-Pacific Biodiversity 9(3): 355-382. https://doi.org/10.1016/j. japb.2016.06.004

Kalawate, A.S. (2018). On a collection of moths (Lepidoptera: Heterocera) from the northern Western Ghats of Maharashtra, India. Zoology and Ecology 28(3): 231-251.

Kirti, J.S. \& N. Singh (2015). Arctiid Moths of India. Vol 1. Nature Books India, New Delhi, 205pp.

Kirti, J.S. \& J.S. Sodhi (2002). Studies on Footman Moths of Sikkim. Journal of Hill Research 15(1): 26-31.

Kirti, J.S. \& J.S. Sodhi (2003). Inventory of tiger moths of Sikkim (Arctiinae: Arctiidae: Lepidoptera). Zoos' Print Journal 18(7): 11431146. https://doi.org/10.11609/JoTT.ZPJ.18.7.1143-6

Kirti, J.S., N. Singh \& R. Joshi (2014). Two new genera of Lithosiin (Lepidoptera: Erebidae: Arctiinae). Tinea 23(1): 41-46.

Kumar, P., M.C. Sharma, R. Saini, \& G.K. Singh (2020). Climatic variability at Gangtok and Tadong weather observatories in Sikkim, India, during 1961-2017. Scientific Reports 10(1): 1-12. https://doi. org/10.1038/s41598-020-71163-y

Mishra, S.B., R.N. Kencharaddi, G.M. Devagiri \& A.K. Khaple (2016). Moths diversity of Kodagu district in Central Western Ghats of Karnataka, India. Indian Journal of Ecology 43(2): 713-718.

Nuss, M., B. Landry, R. Mally, F. Vegliante, A. Tränkner, F. Bauer, J. Hayden, A. Segerer, R. Schouten, H. Li, T. Trofimova, M.A. Solis, J. De Prins \& W. Speidel (2003-2021). Global Information System on
Pyraloidea. www.pyraloidea.org.

Ratnasingham, S. \& P.D. Hebert (2007). BOLD: The Barcode of Life Data System (http://www.barcodinglife.org). Molecular Ecology notes 7(3): 355-364. https://doi.org/10.1111/j.14718286.2006.01687.x

Scoble, M.J. (1992). The Lepidoptera. Form, function and diversity. Oxford University Press, New York, 404pp.

Shubhalaxmi, V. (2018). Field Guide to Indian Moths. Birdwing Publishers, Navi Mumbai, India, 460pp.

Sondhi, Y. \& S. Sondhi (2016). A partial checklist of moths (Lepidoptera) of Dehradun, Mussoorie, and Devalsari in Garhwal, Uttarakhand, India. Journal of Threatened Taxa 8(5): 8756-8776. https://doi. org/10.11609/jott.2814.8.5.8756-8776

Tamang, J.P., M.P. Thapa, R.M. Sharma, A.K. Rai, P. Rai \& R. Dhakal (2005). Carrying capacity study of Teesta Basin in Sikkim. Biological Environment Food Resource 2005, 8.

Uniyal, V.P., P. Dey \& A.K. Sanyal (2016). Diversity of moths Lepidoptera: Heterocera and their potential role as a conservation tool in different protected areas of Uttarakhand. Wildlife Institute of India, Dehradun (DST Project Completion Report), 117pp.

van Nieukerken E.J., L. Kaila, I.J. Kitching, N.P. Kristensen, D.C. Lees, J. Minet, C. Mitter, M. Mutanen, J.C. Regier, T.J. Simonsen, N. Wahlberg, S.H. Yen, R. Zahiri, D. Adamski, J. Baixeras, D. Bartsch, B.A. Bengtsson, J.W. Brown, S.R. Bucheli, D.R. Davis, J.D. Prins, W.D. Prins, M.E. Epstein, P.G. Poole, C. Gielis, P. Hattenschwiler, A. Hausmann, J.D. Holloway, A. Kallies, O. Karsholt, A.Y.Kawahara, S. Koster, M.V. Kozlov, J.D. Lafontaine, G. Lamas, J.F. Landry, S. Lee, M. Nuss, K.T. Park, C. Penz, J. Rota, A. Schintlmeister, B.C. Schmidt, J.C. Sohn, M.A. Solis, G.M. Tarmann, A.D. Warren, S. Weller, R.V. Yakovlev, V.V. Zolotuhin, A. Zwick (2011). Order Lepidoptera Linnaeus, 1758. In: Zhang, Z.Q. (Ed.). Animal Biodiversity: An Outline of Higher-level Classification and Survey of Taxonomic Richness. Zootaxa 3148: 212-221. https://doi.org/10.11646/ zootaxa.3148.1.41

Vattakaven, T., R.M. George, D. Balasubramanian, M. RéjouMéchain, G. Muthusankar, B. R. Ramesh \& R. Prabhakar (2016). India Biodiversity Portal: an integrated, interactive and participatory biodiversity informatics platform. Biodiversity Data Journal 4: e10279. https://doi.org/10.3897/BDJ.4.e10279

Volynkin, A.V. \& K. Černý (2019). A review of the Barsine phaeodonta (Hampson, 1911) species-group, with descriptions of four new species from Indochina and India (Lepidoptera, Erebidae, Arctiinae). Ecologica Montenegrina 21: 70-79. https://doi. org/10.37828/em.2019.21.8

Walker, F. (1866). List of the Specimens of Lepidopterous Insects in the Collection of the British Museum, London 35: 1-321. 

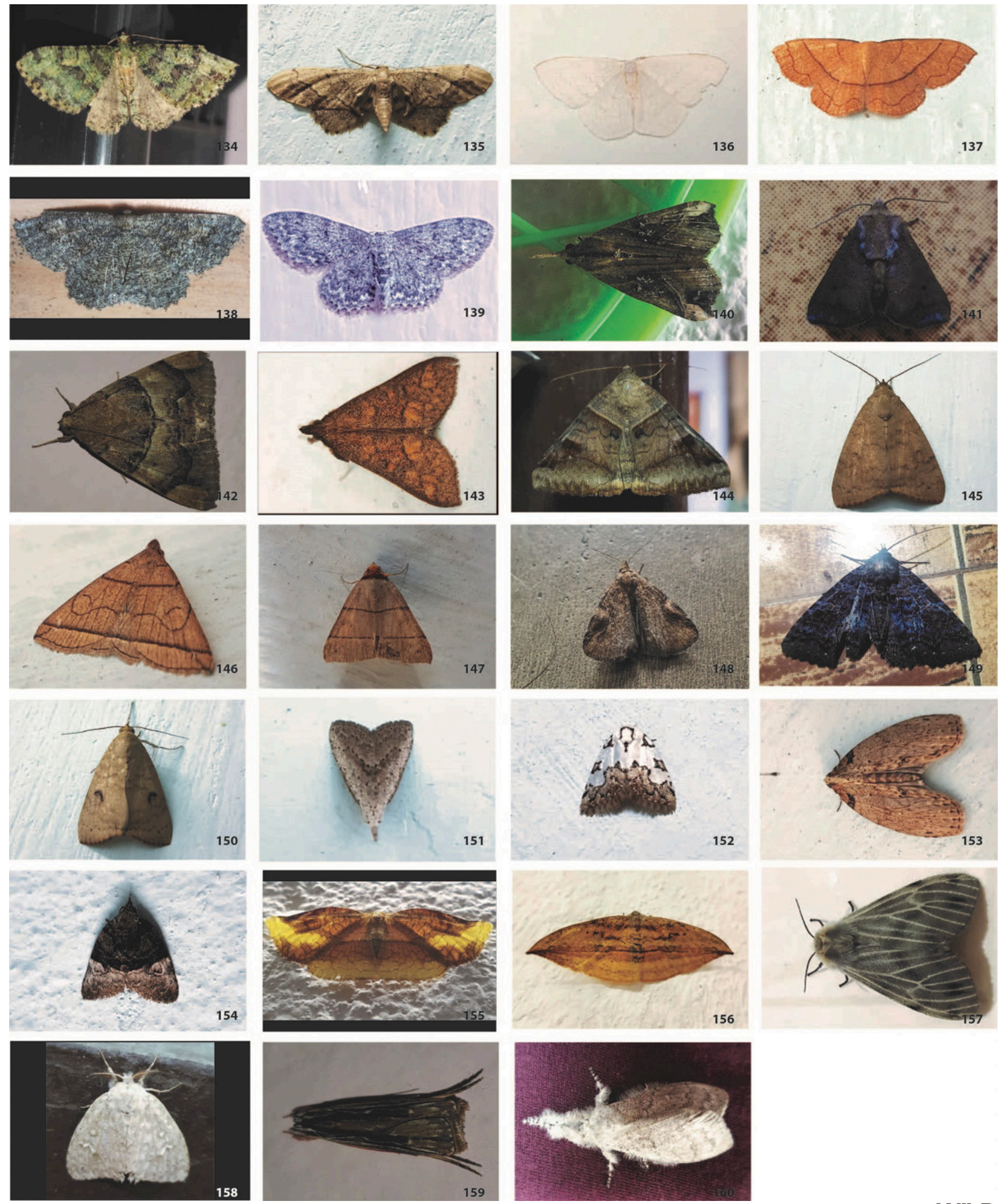

Images 134-160. Photographs of unidentified moths. 
Dr. Albert G. Orr, Griffith University, Nathan, Australia

Dr. Sameer Padhye, Katholieke Universiteit Leuven, Belgium

Dr. Nancy van der Poorten, Toronto, Canada

Dr. Kareen Schnabel, NIWA, Wellington, New Zealand

Dr. R.M. Sharma, (Retd.) Scientist, Zoological Survey of India, Pune, India

Dr. Manju Siliwal, WILD, Coimbatore, Tamil Nadu, India

Dr. G.P. Sinha, Botanical Survey of India, Allahabad, India

Dr. K.A. Subramanian, Zoological Survey of India, New Alipore, Kolkata, India

Dr. P.M. Sureshan, Zoological Survey of India, Kozhikode, Kerala, India

Dr. R. Varatharajan, Manipur University, Imphal, Manipur, India

Dr. Eduard Vives, Museu de Ciències Naturals de Barcelona, Terrassa, Spain

Dr. James Young, Hong Kong Lepidopterists' Society, Hong Kong

Dr. R. Sundararaj, Institute of Wood Science \& Technology, Bengaluru, India

Dr. M. Nithyanandan, Environmental Department, La Ala Al Kuwait Real Estate. Co. K.S.C., Kuwait

Dr. Himender Bharti, Punjabi University, Punjab, India

Mr. Purnendu Roy, London, UK

Dr. Saito Motoki, The Butterfly Society of Japan, Tokyo, Japan

Dr. Sanjay Sondhi, TITLI TRUST, Kalpavriksh, Dehradun, India

Dr. Nguyen Thi Phuong Lien, Vietnam Academy of Science and Technology, Hanoi, Vietnam

Dr. Nitin Kulkarni, Tropical Research Institute, Jabalpur, India

Dr. Robin Wen Jiang Ngiam, National Parks Board, Singapore

Dr. Lional Monod, Natural History Museum of Geneva, Genève, Switzerland.

Dr. Asheesh Shivam, Nehru Gram Bharti University, Allahabad, India

Dr. Rosana Moreira da Rocha, Universidade Federal do Paraná, Curitiba, Brasil

Dr. Kurt R. Arnold, North Dakota State University, Saxony, Germany

Dr. James M. Carpenter, American Museum of Natural History, New York, USA

Dr. David M. Claborn, Missouri State University, Springfield, USA

Dr. Kareen Schnabel, Marine Biologist, Wellington, New Zealand

Dr. Amazonas Chagas Júnior, Universidade Federal de Mato Grosso, Cuiabá, Brasil

Mr. Monsoon Jyoti Gogoi, Assam University, Silchar, Assam, India

Dr. Heo Chong Chin, Universiti Teknologi MARA (UiTM), Selangor, Malaysia

Dr. R.J. Shiel, University of Adelaide, SA 5005, Australia

Dr. Siddharth Kulkarni, The George Washington University, Washington, USA

Dr. Priyadarsanan Dharma Rajan, ATREE, Bengaluru, India

Dr. Phil Alderslade, CSIRO Marine And Atmospheric Research, Hobart, Australia

Dr. John E.N. Veron, Coral Reef Research, Townsville, Australia

Dr. Daniel Whitmore, State Museum of Natural History Stuttgart, Rosenstein, Germany.

Dr. Yu-Feng Hsu, National Taiwan Normal University, Taipei City, Taiwan

Dr. Keith V. Wolfe, Antioch, California, USA

Dr. Siddharth Kulkarni, The Hormiga Lab, The George Washington University, Washington,

D.C., USA

Dr. Tomas Ditrich, Faculty of Education, University of South Bohemia in Ceske

Budejovice, Czech Republic

Dr. Mihaly Foldvari, Natural History Museum, University of Oslo, Norway

Dr. V.P. Uniyal, Wildlife Institute of India, Dehradun, Uttarakhand 248001, India

Dr. John T.D. Caleb, Zoological Survey of India, Kolkata, West Bengal, India

Dr. Priyadarsanan Dharma Rajan, Ashoka Trust for Research in Ecology and the Environment (ATREE), Royal Enclave, Bangalore, Karnataka, India

\section{Fishes}

Dr. Neelesh Dahanukar, IISER, Pune, Maharashtra, India

Dr. Topiltzin Contreras MacBeath, Universidad Autónoma del estado de Morelos, México

Dr. Heok Hee Ng, National University of Singapore, Science Drive, Singapore

Dr. Rajeev Raghavan, St. Albert's College, Kochi, Kerala, India

Dr. Robert D. Sluka, Chiltern Gateway Project, A Rocha UK, Southall, Middlesex, UK

Dr. E. Vivekanandan, Central Marine Fisheries Research Institute, Chennai, India

Dr. Davor Zanella, University of Zagreb, Zagreb, Croatia

Dr. A. Biju Kumar, University of Kerala, Thiruvananthapuram, Kerala, India

Dr. Akhilesh K.V., ICAR-Central Marine Fisheries Research Institute, Mumbai Research

Centre, Mumbai, Maharashtra, India

Dr. J.A. Johnson, Wildlife Institute of India, Dehradun, Uttarakhand, India

\section{Amphibians}

Dr. Sushil K. Dutta, Indian Institute of Science, Bengaluru, Karnataka, India

Dr. Annemarie Ohler, Muséum national d'Histoire naturelle, Paris, France

\section{Reptiles}

Dr. Gernot Vogel, Heidelberg, Germany

Dr. Raju Vyas, Vadodara, Gujarat, India

Dr. Pritpal S. Soorae, Environment Agency, Abu Dubai, UAE.

Prof. Dr. Wayne J. Fuller, Near East University, Mersin, Turkey

Prof. Chandrashekher U. Rivonker, Goa University, Taleigao Plateau, Goa. India

Dr. S.R. Ganesh, Chennai Snake Park, Chennai, Tamil Nadu, India

Dr. Himansu Sekhar Das, Terrestrial \& Marine Biodiversity, Abu Dhabi, UAE
Birds

Dr. Hem Sagar Baral, Charles Sturt University, NSW Australia

Dr. Chris Bowden, Royal Society for the Protection of Birds, Sandy, UK

Dr. Priya Davidar, Pondicherry University, Kalapet, Puducherry, India

Dr. J.W. Duckworth, IUCN SSC, Bath, UK

Dr. Rajah Jayapal, SACON, Coimbatore, Tamil Nadu, India

Dr. Rajiv S. Kalsi, M.L.N. College, Yamuna Nagar, Haryana, India

Dr. V. Santharam, Rishi Valley Education Centre, Chittoor Dt., Andhra Pradesh, India

Dr. S. Balachandran, Bombay Natural History Society, Mumbai, India

Mr. J. Praveen, Bengaluru, India

Dr. C. Srinivasulu, Osmania University, Hyderabad, India

Dr. K.S. Gopi Sundar, International Crane Foundation, Baraboo, USA

Dr. Gombobaatar Sundev, Professor of Ornithology, Ulaanbaatar, Mongolia

Prof. Reuven Yosef, International Birding \& Research Centre, Eilat, Israel

Dr. Taej Mundkur, Wetlands International, Wageningen, The Netherlands

Dr. Carol Inskipp, Bishop Auckland Co., Durham, UK

Dr. Tim Inskipp, Bishop Auckland Co, Durham, UK

Dr. V. Gokula, National College, Tiruchirappalli, Tamil Nadu, India

Dr. Arkady Lelej, Russian Academy of Sciences, Vladivostok, Russia

Dr. Simon Dowell, Science Director, Chester Zoo, UK

Dr. Mário Gabriel Santiago dos Santos, Universidade de Trás-os-Montes e Alto Douro,

Quinta de Prados, Vila Real, Portugal

Dr. Grant Connette, Smithsonian Institution, Royal, VA, USA

Dr. M. Zafar-ul Islam, Prince Saud Al Faisal Wildlife Research Center, Taif, Saudi Arabia

Mammals

Dr. Giovanni Amori, CNR - Institute of Ecosystem Studies, Rome, Italy

Dr. Anwaruddin Chowdhury, Guwahati, India

Dr. David Mallon, Zoological Society of London, UK

Dr. Shomita Mukherjee, SACON, Coimbatore, Tamil Nadu, India

Dr. Angie Appel, Wild Cat Network, Germany

Dr. P.O. Nameer, Kerala Agricultural University, Thrissur, Kerala, India

Dr. Ian Redmond, UNEP Convention on Migratory Species, Lansdown, UK

Dr. Heidi S. Riddle, Riddle's Elephant and Wildlife Sanctuary, Arkansas, USA

Dr. Karin Schwartz, George Mason University, Fairfax, Virginia.

Dr. Lala A.K. Singh, Bhubaneswar, Orissa, India

Dr. Mewa Singh, Mysore University, Mysore, India

Dr. Paul Racey, University of Exeter, Devon, UK

Dr. Honnavalli N. Kumara, SACON, Anaikatty P.O., Coimbatore, Tamil Nadu, India

Dr. Nishith Dharaiya, HNG University, Patan, Gujarat, India

Dr. Spartaco Gippoliti, Socio Onorario Società Italiana per la Storia della Fauna "Giuseppe

Altobello", Rome, Italy

Dr. Justus Joshua, Green Future Foundation, Tiruchirapalli, Tamil Nadu, India

Dr. H. Raghuram, The American College, Madurai, Tamil Nadu, India

Dr. Paul Bates, Harison Institute, Kent, UK

Dr. Jim Sanderson, Small Wild Cat Conservation Foundation, Hartford, USA

Dr. Dan Challender, University of Kent, Canterbury, UK

Dr. David Mallon, Manchester Metropolitan University, Derbyshire, UK

Dr. Brian L. Cypher, California State University-Stanislaus, Bakersfield, CA

Dr. S.S. Talmale, Zoological Survey of India, Pune, Maharashtra, India

Prof. Karan Bahadur Shah, Budhanilakantha Municipality, Kathmandu, Nepal

Dr. Susan Cheyne, Borneo Nature Foundation International, Palangkaraja, Indonesia

Dr. Hemanta Kafley, Wildlife Sciences, Tarleton State University, Texas, USA

\section{Other Disciplines}

Dr. Aniruddha Belsare, Columbia MO 65203, USA (Veterinary)

Dr. Mandar S. Paingankar, University of Pune, Pune, Maharashtra, India (Molecular)

Dr. Jack Tordoff, Critical Ecosystem Partnership Fund, Arlington, USA (Communities)

Dr. Ulrike Streicher, University of Oregon, Eugene, USA (Veterinary)

Dr. Hari Balasubramanian, EcoAdvisors, Nova Scotia, Canada (Communities)

Dr. Rayanna Hellem Santos Bezerra, Universidade Federal de Sergipe, São Cristóvão, Brazil

Dr. Jamie R. Wood, Landcare Research, Canterbury, New Zealand

Dr. Wendy Collinson-Jonker, Endangered Wildlife Trust, Gauteng, South Africa

Dr. Rajeshkumar G. Jani, Anand Agricultural University, Anand, Gujarat, India

Dr. O.N. Tiwari, Senior Scientist, ICAR-Indian Agricultural Research Institute (IARI), New

Delhi, India

Dr. L.D. Singla, Guru Angad Dev Veterinary and Animal Sciences University, Ludhiana, India

Dr. Rupika S. Rajakaruna, University of Peradeniya, Peradeniya, Sri Lanka

Dr. Bahar Baviskar, Wild-CER, Nagpur, Maharashtra 440013, India

Reviewers 2018-2020

Due to pausity of space, the list of reviewers for 2018-2020 is available online.
The opinions expressed by the authors do not reflect the views of the Journal of Threatened Taxa, Wildlife Information Liaison Development Society, Zoo Outreach Organization, or any of the partners. The journal, the publisher, the host, and the partners are not responsible for the accuracy of the political boundaries shown in the maps by the authors.

\footnotetext{
Print copies of the Journal are available at cost. Write to:

The Managing Editor, JoTT,

c/o Wildlife Information Liaison Development Society,

No. 12, Thiruvannamalai Nagar, Saravanampatti - Kalapatti Road,

Saravanampatti, Coimbatore, Tamil Nadu 641035, India

ravi@threatenedtaxa.org
} 


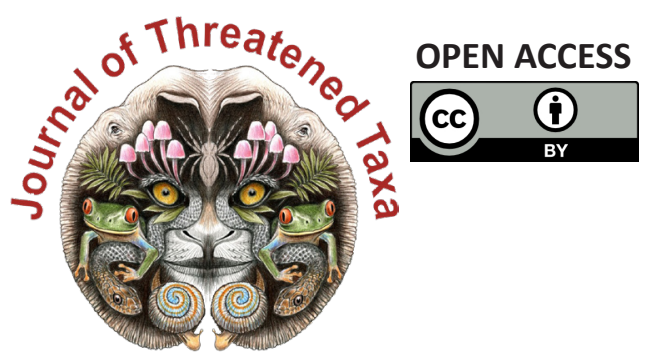

www.threatenedtaxa.org

The Journal of Threatened Taxa (JoTT) is dedicated to building evidence for conservation globally by publishing peer-reviewed articles online every month at a reasonably rapid rate at www.threatenedtaxa.org. All articles published in JoTT are registered under Creative Commons Attribution 4.0 International License unless otherwise mentioned. JoTT allows allows unrestricted use, reproduction, and distribution of articles in any medium by providing adequate credit to the author(s) and the source of publication.

\section{ISSN $0974-7907$ (Online) | ISSN $0974-7893$ (Print)}

\section{October 2021 | Vol. 13 | No. 12 | Pages: 19675-19886 \\ Date of Publication: 26 October 2021 (Online \& Print) DOI: 10.11609/jott.2021.13.12.19675-19886}

Articles

Roosting habits and habitats of the Indian Flying Fox Pteropus medius Temminck, 1825 in the northern districts of Tamil Nadu, India

- M. Pandian \& S. Suresh, Pp. 19675-19688

Diversity and distribution of avifauna at Warathenna-Hakkinda Environmental Protection Area in Kandy, Sri Lanka

- Dinelka Thilakarathne, Tithira Lakkana, Gayan Hirimuthugoda, Chaminda Wijesundara \& Shalika Kumburegama, Pp. 19689-19701

Grass species composition in tropical forest of southern India

- M. Ashokkumar, S. Swaminathan \& R. Nagarajan, Pp. 19702-19713

\section{Communications}

Habitat use and conservation threats to Wild Water Buffalo Bubalus arnee (Mammalia: Artiodactyla: Bovidae) in Koshi Tappu Wildlife Reserve, Nepal

- Reeta Khulal, Bijaya Neupane, Bijaya Dhami, Siddhartha Regmi, Ganesh Prasad Tiwari \& Manita Parajuli, Pp. 19714-19724

Get my head around owls: people perception and knowledge about owls of Andaman Islands

- Shanmugavel Sureshmarimuthu, Santhanakrishnan Babu, Nagaraj Rajeshkumar \& Honnavalli Nagaraj Kumara, Pp. 19725-19732

Abundance and diversity of threatened birds in Nangal Wetland, Punjab, India - Rajwinder Kaur \& Onkar Singh Brraich, Pp. 19733-19742

Evaluation of fish diversity and abundance in the Kabul River with comparisons between reaches above and below Kabul City, Afghanistan

- Ugyen Kelzang, Ahmad Farid Habibi \& Ryan J. Thoni, Pp. 19743-19752

New record of Myrmarachne melanocephala MacLeay, 1839 (Araneae: Salticidae) from Jharkhand, India and biogeographical implications of the co-occurrence of its ant model Tetraponera rufonigra Jerdon, 1851

- Rahul Kumar, Mirtunjay Sharma \& Ajay Kumar Sharma, Pp. 19753-19761

Diversity of spiders (Arachnida: Araneae) and the impact of pruning in Indian sandalwood plantations from Karnataka, India

-S. Padma 1 \& R. Sundararaj, Pp. 19762-19772

New records of cheilostome Bryozoa from the eastern coast of India encrusting on the exoskeleton of live horseshoe crabs of Indian Sundarbans

- Swati Das, Maria Susan Sanjay, Basudev Tripathy, C. Venkatraman \& K.A. Subramanian, Pp. 19773-19780

On the pteridophytes of Bherjan-Borajan-Padumoni Wildlife Sanctuary, Assam, India - Pranjal Borah \& Jayanta Barukial, Pp. 19781-19790

Population status of Heritiera fomes Buch.-Ham., a threatened species from Mahanadi Mangrove Wetland, India

- Sudam Charan Sahu, Manas Ranjan Mohanta \& N.H. Ravindranath, Pp. 19791-19798

Additions to the lichenized and lichenicolous fungi of Jammu \& Kashmir from Kishtwar High Altitude National Park

- Vishal Kumar, Yash Pal Sharma, Siljo Joseph, Roshinikumar Ngangom \& Sanjeeva Nayaka, Pp. 19799-19807

\section{Short Communications}

Is release of rehabilitated wildlife with embedded lead ammunition advisable? Plumbism in a Jaguar Panthera Onca (Mammalia: Carnivora: Felidae), survivor of gunshot wounds - Eduardo A. Díaz, Carolina Sáenz, E. Santiago Jiménez, David A. Egas \& Kelly Swing, Pp. 19808-19812

New record of the Sewing Needle Zipper Loach Paracanthocobitis linypha Singer \& Page, 2015 (Teleostei: Cypriniformes: Nemacheilidae) from the Chindwin drainage of Manipur, India

- Yumnam Rameshori, Yengkhom Chinglemba \& Waikhom Vishwanath, Pp. 19813-19817

Field identification characters to diagnose Microhyla mukhlesuri from closely related M. mymensinghensis (Amphibia: Microhylidae) and range extension of $M$. mukhlesuri up to West Bengal State, India

- Suman Pratihar \& Kaushik Deuti, Pp. 19818-19823
First report of Scipinia horrida (Stål) (Heteroptera: Reduviidae) from Assam, with comments on related genus Irantha Stål

- Anjana Singha Naorem, Santana Saikia, Anandita Buragohain, Rubina Azmeera Begum, Swapnil S. Boyane \& Hemant V. Ghate, Pp. 19824-19830

Flesh fly (Diptera: Sarcophagidae): male terminalia, diversity and expanded geographical distribution from India

- Kanholi Sreejith, Shuvra Kanti Sinha, Santanu Mahato \& Edamana Pushpalatha, Pp. 1983119836

Checklist of moths (Heterocera) of Tadong, Sikkim, India

- Prayash Chettri, Yuki Matsui, Hideshi Naka \& Archana Tiwari, Pp. 19837-19848

New distribution records of Begonia L., B. murina Craib and B. poilanei Kiew (Begoniaceae: Cucurbitales) for Laos

- Phongphayboun Phonepaseuth, Phetlasy Souladeth, Soulivanh Lanorsavanh, Shuichiro Tagane, Thyraphon Vongthavone \& Keooudone Souvannakhoummane Pp. 19849-19854

Notes

A recent sighting of the Stripe-backed Weasel Mustela strigidorsa (Mammalia: Carnivora: Mustelidae) in Hkakabo Razi Landscape, Myanmar

- Sai Sein Lin Oo, Tun Tun, Kyaw Myo Naing \& Paul Jeremy James Bates, Pp. 19855-19859

Are the uplifted reef beds in North Andaman letting nesting Olive Ridley Sea Turtle Lepidochelys olivacea stranded?

- Nehru Prabakaran, Anoop Raj Singh \& Vedagiri Thirumurugan, Pp. 19860-19863

First record of the orb-weaving spider Araneus tubabdominus Zhu \& Zhang, 1993 (Araneae: Araneidae) from India

- Souvik Sen, John T.D. Caleb \& Shelley Acharya, Pp. 19864-19866

The genus Catapiestus Perty, 1831 (Coleoptera: Tenebrionidae: Cnodalonini) from Arunachal Pradesh with one new record to India

- V.D. Hegde \& Sarita Yadav, Pp. 19867-19869

Rediscovery and extended distribution of Indigofera santapaui Sanjappa (Leguminosae: Papilionoideae) from the states of Maharashtra and Gujarat, India

- Kumar Vinod Chhotupuri Gosavi, Sanjay Gajanan Auti, Sharad Suresh Kambale \& Munivenkatappa Sanjappa, Pp. 19870-19873

Additional distribution records of Ceropegia anjanerica, an endemic and 'Endangered' lantern flower of the northern Western Ghats, India

- Samir Shrikant Maity, Ajay Natha Gangurde, Sharad Suresh Kambale, Avinash Ramchandra Gholave, Avinash Asraji Adsul, Ganesh Babaso Pawar \& Kumar Vinod Chhotupuri Gosavi, Pp. 19874-19877

Notes on the extended distribution of Impatiens megamalayana, a recently described balsam in Western Ghats, India

- Anoop P. Balan \& A.J. Robi, Pp. 19878-19883

Book Review

A look over on the scented tree of India (Santalum album - S. Suresh Ramanan \& A. Arunachalam, Pp. 19884-19886
Publisher \& Host
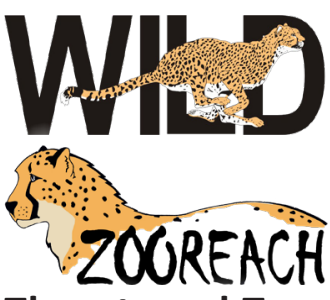

Threatened Taxa 